\title{
An Unconventional Approach to the Enantioselective Synthesis of Caryophylloids \\ Supporing Information
}

\author{
Oleg V. Larionov, E. J. Corey* \\ Department of Chemistry and Chemical Biology, Harvard University, 12 Oxford Street \\ Cambridge, Massachusetts 02138
}

\section{Supporting Information}

1. Materials and Methods. Unless stated otherwise, reactions were performed in flame-dried glassware under a positive pressure of argon using freshly distilled dry solvents. Commercial grade reagents and solvents were used without further purification except as indicated below. $\mathrm{MeOH}$ was distilled over $\mathrm{CaSO}_{4}$. Dichloromethane and 1,2-dichloroethane were distilled from calcium hydride. Toluene, $\mathrm{Et}_{2} \mathrm{O}$ and $\mathrm{THF}$ were purified by Seco Solvent Systems. Diketone $5,{ }^{1}$ silyl ketene acetal $10,{ }^{2}$ a ca. $0.8 \mathrm{M}$ solution of formaldehyde ${ }^{3}$ in THF (storable at $-75^{\circ} \mathrm{C}$ under exclusion of moisture for 2 weeks) and methylenetriphenylphosphorane ${ }^{4}$ were prepared as reported elsewhere. Thin-layer chromatography (TLC) was performed using E. Merck silica gel 60 F254 precoated plates $(0.25 \mathrm{~mm})$. Flash chromatography was performed using Baker silica gel (40 $\mu \mathrm{m}$ particle size). ${ }^{1} \mathrm{H}$ NMR spectra were recorded on Varian Mercury 400 (400 MHz) or Unity/INOVA 500 (500 MHz) spectrometers and chemical shifts are reported in ppm from tetramethylsilane with the solvent resonance as internal standard $(\delta$ $7.26 \mathrm{ppm}$ for $\mathrm{CDCl} 3) .{ }^{13} \mathrm{C}$ NMR spectra were recorded on Varian Mercury $400(100 \mathrm{MHz})$ or Unity/INOVA $500(126 \mathrm{MHz})$ spectrometers with proton decoupling. Chemical shifts are reported in ppm from tetramethylsilane with the solvent as internal $\left(\delta 77.16 \mathrm{ppm}\right.$ for $\left.\mathrm{CDCl}_{3}\right)$. IR spectra were recorded on Avatar 360 FT-IR spectrometer. Low-resolution and highresolution mass spectral analyses were performed at the Harvard University Mass Spectrometry Center. Optical rotations were measured with a Perkin-Elmer 241 polarimeter at the indicated temperature with a sodium lamp (D line, $589 \mathrm{~nm}$ ). Melting points (m.p.) are uncorrected and were recorded on a Büchi capillary melting point apparatus. 


\section{(3aR,5S,7aR)-3a,5-Dihydroxy-7a-methyloctahydro-(1H)-inden-1-one ${ }^{5}$ (6)}

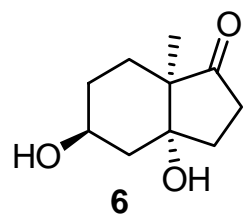

The reaction mixture obtained in the $(R)$-proline-catalyzed Hajos-Parish cyclization $^{1}$ of 2-methyl-2-(3-oxobutyl)-1,3-cyclopentanedione (7 g, 38.5 mmol) was transferred into a $500 \mathrm{~mL}$ one-necked flask and diluted with glacial acetic acid $(100 \mathrm{~mL})$. The mixture was cooled to $-10{ }^{\circ} \mathrm{C}$ and $\mathrm{NaBH}_{4}$ $(0.9 \mathrm{~g}, 23.7 \mathrm{mmol})$ was added in 6 portions within $20 \mathrm{~min}$. The reaction mixture was stirred at $-10{ }^{\circ} \mathrm{C}$ for a further $20 \mathrm{~min}$, then quenched with $1 \mathrm{M}$ aqueous $\mathrm{HCl}(1$ $\mathrm{mL}$ ), and the solvents were distilled off in high vacuum at $55^{\circ} \mathrm{C}$ into a flask placed in a Dewar vessel with liquid $\mathrm{N}_{2}$. The brown residue was loaded onto a silica gel column, and the product was secured by elution with a $\mathrm{CH}_{2} \mathrm{Cl}_{2}-\mathrm{MeOH}$ mixture $(100: 1$ to $10: 1)$ to give the desired $\alpha$-isomer 6 (6.1 g, 86\% over 2 steps) as a colorless oil, as well as the $\beta$-isomeric byproduct $\left(0.4\right.$ g). $\alpha$-isomer 6: $\mathrm{R}_{f} 0.33\left(\mathrm{CH}_{2} \mathrm{Cl}_{2} / \mathrm{MeOH} 20\right.$ : 1). $\beta$-isomer: $\mathrm{R}_{f} 0.4\left(\mathrm{CH}_{2} \mathrm{Cl}_{2} /\right.$ $\mathrm{MeOH} 20: 1)$.

$[\alpha]_{\mathrm{D}}-73$ (c 1.0 in $\left.\mathrm{MeOH}\right) .-{ }^{1} \mathrm{H}$ NMR (400 MHz): 0.98 (s, $\left.3 \mathrm{H}\right), 1.08-1.29(1 \mathrm{H}, \mathrm{m}), 1.30$ $(1 \mathrm{H}, \mathrm{ddd}, J=4.0,10.6,13.8 \mathrm{~Hz}), 1.39(1 \mathrm{H}, \mathrm{dd}, J=9.5,13.8 \mathrm{~Hz}), 1.70-1.80(1 \mathrm{H}, \mathrm{m}), 1.85-$ $2.21(4 \mathrm{H}, \mathrm{m}), 2.26$ (1 H, td, $J=8.2,19.5 \mathrm{~Hz}), 2.48$ (1 H, ddd, $J=3.9,10.3,19.5 \mathrm{~Hz}), 3.92$ (1 H, ddd, $J=5.2,9.2 \mathrm{~Hz}$ ) ppm. $-{ }^{13} \mathrm{C}$ NMR (126 MHz): 17.4, 27.3, 31.1, 33.0, 34.4, 44.0, 52.6, 66.0, 78.5, 220.2 ppm. - IR: $3429 \mathrm{~cm}^{-1}$, 3400, 1722, 1407, 1170, 1092, 1041, 732.

Diol ent-6 was prepared by using (S)-proline as a catalyst in the Hajos-Parish cyclization of 2methyl-2-(3-oxobutyl)-1,3-cyclopentanedione and subsequent reduction with $\mathrm{NaBH}_{4} / \mathrm{AcOH}$. The product had identical spectroscopic data, but the opposite sign of the optical rotation.

(3aR,7aR)-7a-Methyl-3a-(trimethylsilyloxy)-2,3,3a,6,7,7a-hexahydro-(1H)-inden-1-one

(7)

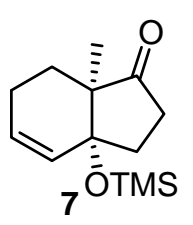

To solution of alcohol 6 (0.9 g, $4.9 \mathrm{mmol})$ and triphenylphosphine $(3.18 \mathrm{~g}$, $11.9 \mathrm{mmol})$ in THF $(60 \mathrm{~mL})$, a solution of diethyl azadicarboxylate (DEAD) $(2.1 \mathrm{~g}, 11.9 \mathrm{mmol})$ in THF $(5 \mathrm{~mL})$ was added dropwise under argon at $-20{ }^{\circ} \mathrm{C}$ within $30 \mathrm{~min}$. The mixture was stirred at $23{ }^{\circ} \mathrm{C}$ for $16 \mathrm{~h}$. Then 1(trimethylsilyl)imidazole $(2.1 \mathrm{~g}, 14.4 \mathrm{mmol})$, and $1 \mathrm{M}$ TBAF solution in THF $(0.54 \mathrm{~mL}, 0.54$ mmol) were added at $0{ }^{\circ} \mathrm{C}$. The reaction mixture was stirred at room temperature for $5 \mathrm{~h}$. 
Then, THF was evaporated under reduced pressure at $30{ }^{\circ} \mathrm{C}$, and the residue was dissolved in toluene and subjected to column chromatography (hexanes/EtOAc) to give ketone 7 (0.71 g, $61 \%$ over 2 steps) as a colorless oil. $\mathrm{R}_{f} 0.45$ (hexane/EtOAc 5:1). $[\alpha]_{\mathrm{D}}{ }^{20}+117$ (c 1.0 in $\left.\mathrm{CHCl}_{3}\right) .-{ }^{1} \mathrm{H}$ NMR $(500 \mathrm{MHz}): 0.11(9 \mathrm{H}, \mathrm{s}), 0.95(3 \mathrm{H}, \mathrm{s}), 1.42(1 \mathrm{H}, \mathrm{dddd}, J=8.5,12.0$, 12.0, 12.0 Hz), $1.76(1 \mathrm{H}, \mathrm{ddd}, J=4.0,7.0,17.0 \mathrm{~Hz}), 1.79-2.01(3 \mathrm{H}, \mathrm{m}), 2.05-2.18(2 \mathrm{H}, \mathrm{m})$, 2.24-2.37 (1 H, m), 5.69 (1 H, ddd, $J=2.0,2.0,12.5 \mathrm{~Hz}), 5.79$ (1 H, ddd, $J=4.0,4.0,12.5$ Hz) ppm. $-{ }^{13} \mathrm{C}$ NMR (126 MHz): 2.4, 18.4, 22.1, 26.0, 34.6, 34.8, 52.4, 77.9, 130.8, 131.7, 220.4 ppm. - IR: $2956 \mathrm{~cm}^{-1}, 1739,1248,1106,904,837,742$. - LRMS (ESI): 256.2 $\left[\mathrm{M}+\mathrm{NH}_{4}{ }^{+}\right]$. - HRMS (ESI): calcd: $256.1727\left[\mathrm{M}+\mathrm{NH}_{4}{ }^{+}\right]$; found: 256.1794.

The ketone ent-7 was prepared in the same manner from ent-6, and had identical spectroscopic data, but the opposite sign of the optical rotation.

\section{(1R,3aR,7aS)-7a-Methyl-2,3,3a,6,7,7a-hexahydro-(1H)-indene-1,3a-diol (9)}

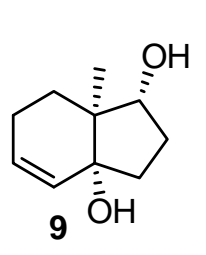

To a solution of $7(1.6 \mathrm{~g}, 6.7 \mathrm{mmol})$ in $\mathrm{THF}(25 \mathrm{~mL})$ at $0{ }^{\circ} \mathrm{C}$ was added a freshly-prepared $0.2 \mathrm{M}$ solution of $(S)$-Bu-CBS reagent $(\mathbf{8})(3.4 \mathrm{~mL}, 0.67 \mathrm{mmol})$ in THF. Then borane-dimethyl sulfide $(0.64 \mathrm{~mL}, 6.7 \mathrm{mmol})$ was added dropwise within $10 \mathrm{~min}$. The reaction mixture was stirred at $0{ }^{\circ} \mathrm{C}$ for $1.5 \mathrm{~h}$, then $\mathrm{MeOH}(2.5 \mathrm{~mL})$ and $10 \%$ aqueous $\mathrm{NaOH}$ solution $(2.5 \mathrm{~mL})$ were introduced slowly. The resulting mixture was diluted with $\mathrm{CH}_{2} \mathrm{Cl}_{2}(30 \mathrm{~mL})$, dried over $\mathrm{Na}_{2} \mathrm{SO}_{4}$ and concentrated under reduced pressure. The residue was purified by column chromatography (hexanes/EtOAc), and the intermediate alcohol was immediately dissolved in THF ( $5 \mathrm{~mL}$ ) and treated with triethylamine trihydrofluoride $(1 \mathrm{~mL}$, Aldrich). The reaction mixture was stirred at $23{ }^{\circ} \mathrm{C}$ for $2 \mathrm{~h}$. It was then concentrated under reduced pressure, and the residue was purified by column chromatography (hexanes/EtOAc) to give diol $9(0.69 \mathrm{~g}, 61 \%) . \mathrm{R}_{f} 0.33$ (hexane/EtOAc 1:1). $[\alpha]_{\mathrm{D}}{ }^{20}+39\left(c 0.3\right.$ in $\left.\mathrm{CHCl}_{3}\right) .-{ }^{1} \mathrm{H}$ NMR (500 MHz): $1.02(3 \mathrm{H}, \mathrm{s}), 1.34$ 1.45 (2 H, m), 1.67 (1 H, br s), 1.76-1.83 (1 H, m), 1.89-1.96 (1 H, m), 1.97-2.02 (1 H, m), 2.06-2.21 (3 H, m), $2.26(1 \mathrm{H}, \mathrm{br}$ s)3.81 (1 H, ddd, $J=2.0,6.0,6.0 \mathrm{~Hz}), 5.61(1 \mathrm{H}, \mathrm{ddd}, J=$ 3.0, 3.0, $10.0 \mathrm{~Hz}), 5.66(1 \mathrm{H}, \mathrm{dd}, J=2.0,10.0 \mathrm{~Hz}) \mathrm{ppm} .-{ }^{13} \mathrm{C} \mathrm{NMR}(126 \mathrm{MHz}): 13.3,22.0$, 29.9, 31.0, 38.4, 47.4, 80.6, 81.0, 125.9, 132.3 ppm. - IR: $3354 \mathrm{~cm}^{-1}, 3019,2935,1375,1023$, 731. - LRMS (ESI): $169.1\left[\mathrm{M}+\mathrm{H}^{+}\right]$. - HRMS (ESI): calcd: $191.1043\left[\mathrm{M}+\mathrm{Na}^{+}\right]$; found: 191.1056. 
The diol ent-9 was prepared in the same manner from ent-7, using $(R)$-Bu-CBS reagent (ent8) as the catalyst on the reduction step. The product showed identical spectroscopic data, but the opposite sign of the optical rotation.

(1R,3aR,7aS)-3a-Hydroxy-7a-methyl-2,3,3a,6,7,7a-hexahydro-(1H)-inden-1-yl 4methylbenzenesulfonate (18)

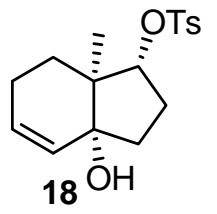

To a solution of $9(0.9 \mathrm{~g}, 5.4 \mathrm{mmol})$ in pyridine $(7 \mathrm{~mL})$ was added $p$ toluenesulfonyl chloride $(1.73 \mathrm{~g}, 9 \mathrm{mmol})$, and the reaction mixture was stirred at $23{ }^{\circ} \mathrm{C}$ for $20 \mathrm{~h}$. The pyridine was then removed in vacuo $(0.05$ mbar), and the residue was purified by column chromatography (hexanes/EtOAc) to give tosylate $18(1.3 \mathrm{~g}, 75 \%)$ as a viscous colorless oil. [ $\alpha]_{\mathrm{D}}{ }^{20}-42$ (c 1.5 in $\left.\mathrm{CHCl}_{3}\right) ;-{ }^{1} \mathrm{H}$ NMR (500 MHz): $0.96(3 \mathrm{H}, \mathrm{s}), 1.33(1 \mathrm{H}, \mathrm{ddd}, J=6.7,6.7,13.5 \mathrm{~Hz}), 1.67$ $(1 \mathrm{H}$, ddd, $J=6: 7,6.7,13.5 \mathrm{~Hz}), 1.65(1 \mathrm{H}, \mathrm{br}$ s), 1.70 (1 H, ddd, $J=3.4,3.4,13.5 \mathrm{~Hz}), 1.75$ $1.87(3 \mathrm{H}, \mathrm{m}), 1.89-2.03(2 \mathrm{H}, \mathrm{m}), 2.43(3 \mathrm{H}, \mathrm{s}), 4.57(1 \mathrm{H}, \mathrm{dd}, J=7.5,7.5 \mathrm{~Hz}), 5.55(1 \mathrm{H}, \mathrm{d}$, $J=10.0 \mathrm{~Hz}), 5.66(1 \mathrm{H}, \mathrm{ddd}, J=3.8,10.0 \mathrm{~Hz}), 7.32(2 \mathrm{H}, \mathrm{d}, J=7.8 \mathrm{~Hz}), 7.77(2 \mathrm{H}, \mathrm{d}, J=7.8$ Hz) ppm. $-{ }^{13} \mathrm{C}$ NMR (126 MHz): 15.6, 21.4, 21.6, 26.7, 27.4, 35.7, 46.1, 85.0, 123.7, 127.7, 128.2, 129.7, 132.2, 134.1, 136.0, 144.6, 149.6 ppm. - IR: $2923 \mathrm{~cm}^{-1}, 1352,1293,1173,954$, 887. - LRMS (ESI): $340.1\left[\mathrm{M}+\mathrm{Na}^{+}\right]$. - HRMS (ESI): calcd: $340.1577\left[\mathrm{M}+\mathrm{NH}_{4}^{+}\right]$; found: 340.1581 .

The tosylate ent-18 was prepared in the same manner from ent-9, and had identical spectroscopic data, but the opposite sign of the optical rotation.

\section{(aS)-6-Methylcyclonona-(2Z,6E)-dienone (4)}

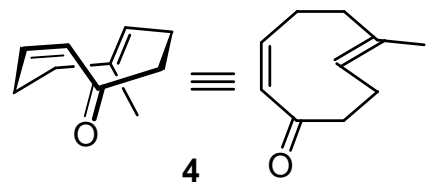

Sodium hydride ( $60 \%$ in oil, $200 \mathrm{mg}, 5 \mathrm{mmol})$ was added in 5 portions within $20 \mathrm{~min}$ to a solution of $\mathbf{1 8}(1.2 \mathrm{~g}, 3.72 \mathrm{mmol})$ in DMF $(20 \mathrm{~mL})$ at $23{ }^{\circ} \mathrm{C}$ The reaction mixture was stirred at $23{ }^{\circ} \mathrm{C}$ for $2 \mathrm{~h}$, then cooled to $0{ }^{\circ} \mathrm{C}$ and quenched by slowly adding cold water $(20 \mathrm{~mL})$. The resulting mixture was extracted with $\mathrm{Et}_{2} \mathrm{O}(4 \times 20 \mathrm{~mL})$. The extracts were dried over $\mathrm{Na}_{2} \mathrm{SO}_{4}$ and concentrated under reduced pressure at $23{ }^{\circ} \mathrm{C}$ The cyclic enone 4 (540 mg, 97\%) was secured by column chromatography (hexanes/ $\mathrm{Et}_{2} \mathrm{O}$ ) as a colorless liquid with a distinct terpene scent. $\mathrm{R}_{f} 0.6$ (hexane/EtOAc 5:1). 4: $[\alpha]_{\mathrm{D}}{ }^{20}+89$ (c 5.0 in $\left.\mathrm{CHCl}_{3}\right) .-{ }^{1} \mathrm{H}$ NMR (500 MHz): $1.57(3 \mathrm{H}$, s), $1.63(1 \mathrm{H}, \mathrm{dd}, J=10.0,12.5 \mathrm{~Hz}), 2.14(1 \mathrm{H}, \mathrm{dd}, J=6.0,7.5 \mathrm{~Hz}), 2.15-2.22(2 \mathrm{H}, \mathrm{m}), 2.28$ 
(1 H, dd, $J=7.0,10.5 \mathrm{~Hz}), 2.36(1 \mathrm{H}, \mathrm{ddd}, J=1.5,10.5,10.5 \mathrm{~Hz}), 2.66(1 \mathrm{H}, \mathrm{qd}, J=7.5,11.5$ $\mathrm{Hz}), 2.85(1 \mathrm{H}, \mathrm{qd}, J=7.5,11.5 \mathrm{~Hz}), 5.42(1 \mathrm{H}, \mathrm{dd}, J=3.0,12.0 \mathrm{~Hz}), 5.60(1 \mathrm{H}, \mathrm{ddd}, J=7.5$, 9.0, 9.0 Hz), $6.21(1 \mathrm{H}, \mathrm{dd}, J=1.5,12.0 \mathrm{~Hz}) \mathrm{ppm} .-{ }^{13} \mathrm{C}$ NMR $(126 \mathrm{MHz}): 16.5,24.1,25.9$, 37.0, 42.8, 127.2, 132.7, 134.6, 136.8, 206.0 ppm. - IR: $2933 \mathrm{~cm}^{-1}, 2858,1687,1653,1452$, 1169, 973, 831, 734. - LRMS (EI): $150\left[\mathrm{M}^{+}\right]$, 135, 107, 93, 79. - HRMS (ESI): calcd: $173.0937\left[\mathrm{M}+\mathrm{Na}^{+}\right]$; found: 173.0937.

The enantiomeric ent-4 $\left([\alpha]_{\mathrm{D}}{ }^{20}-87\left(\right.\right.$ c 5.0 in $\left.\left.\mathrm{CHCl}_{3}\right)\right)$ was prepared from ent-18 following the same procedure, and was spectroscopically identical.

\section{(8R)-(1-Hydroxy-2-methylpropan-2-yl)-5-methylcyclonon-(4E)-enone (16)}

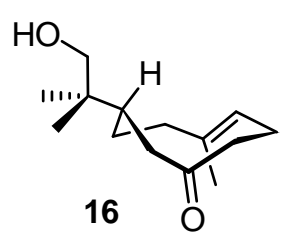

Dienone 4 (200 mg, $1.33 \mathrm{mmol}$ ) and methyl trimethylsilyl dimethylketene acetal (15) (0.47 g, $2.64 \mathrm{mmol})$ were dissolved in dichloromethane $(6 \mathrm{~mL})$ at $23{ }^{\circ} \mathrm{C}$ The solution was then cooled to $-78^{\circ} \mathrm{C}$, and triphenylcarbenium perchlorate (24 $\mathrm{mg}, 0.068 \mathrm{mmol}, 5 \mathrm{~mol} \%$ ) was added. The reaction mixture was stirred at $-78{ }^{\circ} \mathrm{C}$ for $40 \mathrm{~min}$, at which point complete conversion of starting material was determined by TLC analysis. A $1 \mathrm{M}$ solution of DIBAL in hexane $(5 \mathrm{~mL}, 5$ mmol) was added dropwise within $5 \mathrm{~min}$, and the mixture was stirred at $-78{ }^{\circ} \mathrm{C}$ for a further 1h. $50 \%$ aqueous $\mathrm{NaOH}$ solution was added, and the mixture was vigorously stirred at $23{ }^{\circ} \mathrm{C}$ for $2 \mathrm{~h}$, then extracted with dichloromethane $(3 \times 20 \mathrm{~mL})$. The combined organic phases were dried over $\mathrm{Na}_{2} \mathrm{SO}_{4}$ and concentrated under reduced pressure. The crude material was purified by column chromatography (hexanes/EtOAc) to give the alcohol 16 (250 $\mathrm{mg}, 84 \%$ ) as a colorless oil. $[\alpha]_{\mathrm{D}}{ }^{20}+31\left(c 1.5\right.$ in $\left.\mathrm{CHCl}_{3}\right) .-{ }^{1} \mathrm{H}$ NMR (500 MHz): $0.57(3 \mathrm{H}, \mathrm{s}), 1.03(3 \mathrm{H}, \mathrm{s})$, $1.23(1 \mathrm{H}$, dddd, $J=3.5,10.5,13.5,13.5 \mathrm{~Hz}), 1.54(1 \mathrm{H}, \mathrm{ddd}, J=3,3,10.5 \mathrm{~Hz}), 1.75(1 \mathrm{H}$, dd, $J=3.5,3.5 \mathrm{~Hz}), 1.79(3 \mathrm{H}, \mathrm{s}), 1.92(1 \mathrm{H}, \mathrm{ddd}, J=4.0,12.0,12.0 \mathrm{~Hz}), 2.05(1 \mathrm{H}, \mathrm{dd}, J=$ 3.5, $15.5 \mathrm{~Hz}), 2.18-2.31(4 \mathrm{H}, \mathrm{m}), 2.50-2.59(1 \mathrm{H}, \mathrm{m}), 2.82-2.91(1 \mathrm{H}, \mathrm{m}), 3.04(1 \mathrm{H}, \mathrm{dd}, J=$ 11.5, $11.5 \mathrm{~Hz}), 3.52$ ( $1 \mathrm{H}, \mathrm{dd}, J=3.0,12.5 \mathrm{~Hz}), 4.49$ (1 H, dd, $J=4.0,10.5 \mathrm{~Hz}), 5.25(1 \mathrm{H}$, dd, $J=7.0,8.5 \mathrm{~Hz}$ ) ppm. $-{ }^{13} \mathrm{C}$ NMR (126 MHz): 16.5, 18.5, 21.3, 24.3, 30.8, 38.9, 40.6, 42.8, 47.9, 70.3, 121.6, 139.2, 220.4 ppm. - LRMS (ESI): $225.2\left[\mathrm{M}+\mathrm{H}^{+}\right]$. - HRMS (ESI): calcd: $225.1855\left[\mathrm{M}+\mathrm{H}^{+}\right]$; found: 225.1846 . 


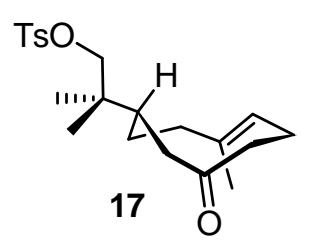

To a solution of $16(80 \mathrm{mg}, 0.36 \mathrm{mmol})$ in pyridine $(1.5 \mathrm{~mL})$ was added p-toluenesulfonyl chloride (115 $\mathrm{mg}, 0.6 \mathrm{mmol})$, and the reaction mixture was stirred at $23{ }^{\circ} \mathrm{C}$ for $12 \mathrm{~h}$. The pyridine was then removed in high vacuum, and the residue was purified by column chromatography (hexane/EtOAc) and then by recrystallization from hexane/2-propanol to give tosylate $17(97 \mathrm{mg}, 71 \%)$ as a colorless solid. $[\alpha]_{\mathrm{D}}{ }^{20}+2.3\left(\right.$ c 2.6 in $\left.\mathrm{CHCl}_{3}\right) .-{ }^{1} \mathrm{H} \mathrm{NMR}$ (500 MHz): 0.88 (6 H, s), 1.08-1.18 (1 H, m), 1.56 (3 H, s), 1.62-1.68 (1 H, m), $1.71(1 \mathrm{H}$, ddd, $J=2.5,7.0,7.0 \mathrm{~Hz}), 1.79(1 \mathrm{H}$, ddd, $J=3.5,12.5,12.5 \mathrm{~Hz}), 1.97(1 \mathrm{H}, \mathrm{dd}, J=7.0,14.0$ Hz), 2.06-2.16 (2 H, m), $2.52(1 \mathrm{H}, \mathrm{dd}, J=3.0,14,0 \mathrm{~Hz}), 2.33-2.42(1 \mathrm{H}, \mathrm{m}), 2.46(3 \mathrm{H}, \mathrm{s})$, 2.49-2.61 (2 H, m), $3.78(2 \mathrm{H}, \mathrm{s}), 5.31(1 \mathrm{H}, \mathrm{dd}, J=5.5,5.5 \mathrm{~Hz}), 7.36(2 \mathrm{H}, \mathrm{d}, J=8.0 \mathrm{~Hz})$, 7.80 ((2 H, d, $J=8.0 \mathrm{~Hz})$ ppm. $-{ }^{13} \mathrm{C}$ NMR (126 MHz): 16.6, 21.6, 22.1, 22.8, 29.9, 37.8, 40.4, 40.7, 42.4, 49.3, 77.3, 123.4, 127.8, 129.8, 132.8, 137.2, 144.7, 220.6 ppm. - IR: 2932 $\mathrm{cm}^{-1}, 1717,1653,1362,1176,962 .-$ LRMS (ESI): 396.25 [M+NH$\left.{ }_{4}^{+}\right] .-$HRMS (ESI): calcd: $379.1943\left[\mathrm{M}+\mathrm{H}^{+}\right]$; found: 379.1942 .

\section{(1S,9R)-6,10,10-Trimethylbicyclo[7.2.0]undec-(5E)-en-2-one ${ }^{6}(19)$}

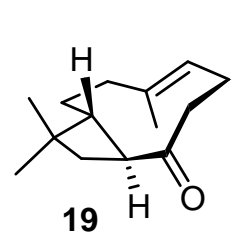

To a solution of tosylate $\mathbf{1 7}(130 \mathrm{mg}, 0.34 \mathrm{mmol})$ in 2-propanol $(2 \mathrm{~mL})$ at $10{ }^{\circ} \mathrm{C}$ was added a $1 \mathrm{M}$ solution of potassium tert-butoxide in tert-butanol $(0.56 \mathrm{mmol}, 0.56 \mathrm{~mL})$, and the reaction mixture was stirred at $0{ }^{\circ} \mathrm{C}$ for 36 h. Saturated aqueous $\mathrm{NH}_{4} \mathrm{Cl}$ solution $(0.5 \mathrm{~mL})$ was added, followed by dichloromethane $(10 \mathrm{~mL})$. The mixture was dried over $\mathrm{Na}_{2} \mathrm{SO}_{4}$ and concentrated under reduced pressure. The crude product was purified by column chromatography (hexanes/EtOAc) to give 15-norcaryophyllen-8-one (19) (52 $\mathrm{mg}, 85 \%$ ) as a viscous oil, slowly solidifying at $23{ }^{\circ} \mathrm{C}[\alpha]_{\mathrm{D}}{ }^{20}-75$ (c 1.1 in $\left.\mathrm{CHCl}_{3}\right)$; lit: ${ }^{6}[\alpha]_{\mathrm{D}}{ }^{20}-71.5$ (c 1.4 in $\left.\mathrm{CHCl}_{3}\right)$; lit: ${ }^{7}[\alpha]_{\mathrm{D}}{ }^{20}-78.7$ (c 1.28 in $\left.\mathrm{CHCl}_{3}\right) .{ }^{1}{ }^{1} \mathrm{H}$ NMR (500 MHz): $0.97(3 \mathrm{H}, \mathrm{s}), 1.01(3 \mathrm{H}, \mathrm{s}), 1.49-$ $1.66(5 \mathrm{H}, \mathrm{m}), 1.75(3 \mathrm{H}, \mathrm{s}), 2.05(1 \mathrm{H}, \mathrm{dd}, J=9.5,11.0 \mathrm{~Hz}), 2.10-2.26$ (3 H, m), 2.36-2.47 (1 $\mathrm{H}, \mathrm{m}), 2.68-2.76(1 \mathrm{H}, \mathrm{m}), 2.83(1 \mathrm{H}, \mathrm{dt}, J=8.0,9.0 \mathrm{~Hz}), 5.24(1 \mathrm{H}, \mathrm{dd}, J=6.5,7.5 \mathrm{~Hz}) \mathrm{ppm}$. $-{ }^{13} \mathrm{C}$ NMR (126 MHz): 15.9, 21.9, 23.1, 28.8, 29.3, 33.4, 35.7, 39.8, 40.7, 51.9, 52.6, 122.7, 138.1, 216.3 ppm. - IR: $2928 \mathrm{~cm}^{-1}, 2855,1694,1449,1159,1091,756$. 


\section{(-)- $\beta$-Caryophyllene (1)}

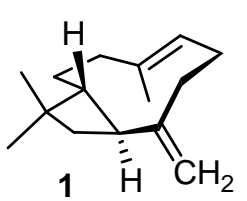

To a suspension of methyltriphenylphosphonium bromide (526 mg, 1.47 mmol) in THF $(3.5 \mathrm{~mL})$ was added dropwise at $0{ }^{\circ} \mathrm{C}$ a $2.5 \mathrm{M}$ solution of $n$ BuLi in hexane $(0.49 \mathrm{~mL}, 1.23 \mathrm{mmol})$. The yellow suspension was stirred for $15 \mathrm{~min}$ at $0{ }^{\circ} \mathrm{C}$, and then at $23{ }^{\circ} \mathrm{C}$ for $30 \mathrm{~min}$. A solution of $19(50 \mathrm{mg}$, $0.24 \mathrm{mmol})$ in THF $(2 \mathrm{~mL})$ was added at $0{ }^{\circ} \mathrm{C}$, and the reaction mixture was stirred at $23{ }^{\circ} \mathrm{C}$ for $1 \mathrm{~h}$. Saturated aqueous $\mathrm{NH}_{4} \mathrm{Cl}$ solution $(0.5 \mathrm{~mL})$ and hexane $(5 \mathrm{~mL})$ were added, and the organic phase was dried with $\mathrm{Na}_{2} \mathrm{SO}_{4}$ and concentrated. The residue was purified by column chromatography (hexanes/EtOAc) to give $\beta$-caryophyllene (1) (39 $\mathrm{mg}, 79 \%$ ) as a colorless oil. $[\alpha]_{\mathrm{D}}{ }^{20}-13$ (c 1.5 in $\mathrm{CHCl}_{3}$ ) lit: ${ }^{8}[\alpha]_{\mathrm{D}}{ }^{20}-12.9$ (c 1.07 in $\left.\mathrm{CHCl}_{3}\right) .-{ }^{1} \mathrm{H}$ NMR $(500 \mathrm{MHz})$ : $0.97(3 \mathrm{H}, \mathrm{s}), 1.00(3 \mathrm{H}, \mathrm{s}), 1.61$ (3 H, br s), 1.43-1.57 (2 H, m), 1.57-1.71 (3 H, m), 1.91 (1 H, ddd, $J=5.0,5.0,12.0 \mathrm{~Hz}), 1.96-2.02(2 \mathrm{H}, \mathrm{m}), 2.08(1 \mathrm{H}, \mathrm{ddd}, J=3.3,3.3,12.3 \mathrm{~Hz})$, 2.17-2.23 (1 H, m), 2.20-2.36 (2 H, m), 4.82 (1 H, br s), 4.94 (1 H, br s), 5.30 (1 H, dd, $J=$ 4.5, 10.0 Hz) ppm. $-{ }^{13} \mathrm{C}$ NMR (126 MHz): 16.3, 22.6, 28.4, 29.4, 32.9, 34.8, 40.0, 40.3, 48.5, 53.6, 111.6, 124.3, 135.2, 154.7 ppm. - IR: $2924 \mathrm{~cm}^{-1}$, 2855, 1699, 1457, 885.

The synthetic 1 is identical with a natural sample by all physical measurements.

\section{Methyl (2S)-[4-methyl-8-oxocyclonon-(4E)-enyl]acetate (11)}

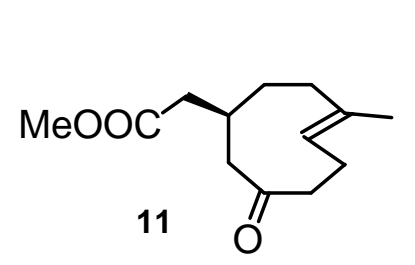

A solution of ent-4 (120 mg, $0.8 \mathrm{mmol})$ and silyl ketene acetal 10 $(0.84 \mathrm{~mL}, 770 \mathrm{mg}, 4.1 \mathrm{mmol})$ in 1,2-dichloroethane $(3.5 \mathrm{~mL})$ was degassed using 3 freeze-thaw-pump cycles, then cooled to $-30{ }^{\circ} \mathrm{C}$ and triphenylcarbenium perchlorate $(82 \mathrm{mg}, 0.24 \mathrm{mmol})$ was added at once. The mixture was stirred for $1 \mathrm{~h}$, as the temperature was slowly rising to $0{ }^{\circ} \mathrm{C}$. Triethylamine $(1 \mathrm{~mL})$ was added, and the solvents were removed at reduced pressure. The residue was redissolved in dichloromethane $(7 \mathrm{~mL})$, and silica gel $(1.5 \mathrm{~g})$ was added. The solvent was removed in vacuo. The solid material was loaded onto the silica gel column, and the product 11 (114 mg, 64\%) was secured by a standard chromatography technique as a colorless oil. $\mathrm{R}_{f} 0.2$ (7:1 hexane/ethyl acetate). $[\alpha]_{\mathrm{D}}{ }^{20}-24\left(\right.$ c 0.25 in $\left.\mathrm{CHCl}_{3}\right) .-{ }^{1} \mathrm{H}_{\mathrm{NMR}}(500$ MHz): 1.30-1.54 (2 H, m), 1.62 (3 H, s), 2.01-2.23 (5 H, m), 2.31-2.40 (2 H, m), 2.43-2.47 (2 H, m), 2.59-2.66 (1H , m), 2.70-2.76 (1 H, m), $3.68(3 \mathrm{H}, \mathrm{s}), 5.41(1 \mathrm{H}, \mathrm{dd}, J=5.5,11.0$ 
Hz) ppm. $-{ }^{13} \mathrm{C}$ NMR (126 MHz): 16.7, 23.2, 33.4, 34.5, 39.8, 40.6, 43.2, 51.5, 53.5, 124.0, 136.8, 172.9, 213.5 ppm. - IR: $2928 \mathrm{~cm}^{-1}, 2857,1734,1700,1456,1260,1154,835,734$. LRMS (ESI): 263.1 [M+K ${ }^{+}$. - HRMS (ESI): calcd: 247.1305 [M+Na ${ }^{+}$; found: 247.1300 .

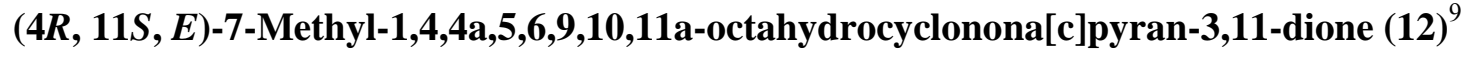

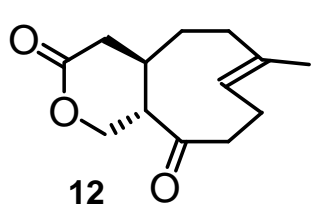

To a solution of $11(180 \mathrm{mg}, 0.8 \mathrm{mmol})$ in $\mathrm{THF}(2 \mathrm{~mL})$ at $-60{ }^{\circ} \mathrm{C}$ was added dropwise a freshly prepared $0.5 \mathrm{M}$ solution of sodium tertpentoxide in THF (1.5 mmol, $3 \mathrm{~mL})$, and the reaction mixture was stirred for $5 \mathrm{~h}$, while the temperature was allowed to slowly climb to $20{ }^{\circ} \mathrm{C}$. The mixture was cooled to $-60^{\circ} \mathrm{C}$ and a ca. $0.8 \mathrm{M}$ solution of formaldehyde in THF $(6$ $\mathrm{mL}, 4.8 \mathrm{mmol}$ ) was injected at once. The mixture was stirred at $-60^{\circ} \mathrm{C}$ for $10 \mathrm{~h}$, then quenched with saturated aqueous $\mathrm{NH}_{4} \mathrm{Cl}$ solution $(1 \mathrm{~mL})$, diluted with dichloromethane (10 $\mathrm{mL}$ ), and dried over $\mathrm{Na}_{2} \mathrm{SO}_{4}$. The solids were filtered off, and the solvents were removed under reduced pressure. The residue was purified by column chromatography, followed by recrystallization from THF - hexane to give $105 \mathrm{mg}(59 \%)$ of the lactone 12 as a colorless solid, as well as unreacted 11 (26 mg). $\mathrm{R}_{f} 0.15$ (2:1 hexane/ethyl acetate). m.p. $162{ }^{\circ} \mathrm{C}$ (lit. ${ }^{9}$ $\left.163{ }^{\circ} \mathrm{C}\right) .[\alpha]_{\mathrm{D}}{ }^{20}+120\left(\right.$ c 0.28 in $\mathrm{CHCl}_{3} ;$ lit. $^{9}[\alpha]_{\mathrm{D}}{ }^{20}+111, c 0.25$ in $\left.\mathrm{CHCl}_{3}\right) .-{ }^{1} \mathrm{H} \mathrm{NMR}$ (500 MHz): 1.56-1.63 (2 H, m), $1.81(3 \mathrm{H}, \mathrm{s}), 2.03-2.12(1 \mathrm{H}, \mathrm{m}), 2.20$ (1 H, ddd, $J=3.0,3.0$, $10.5 \mathrm{~Hz}), 2.22-2.28(1 \mathrm{H}, \mathrm{m}), 2.30-2.37(3 \mathrm{H}, \mathrm{m}), 2.60(1 \mathrm{H}, \mathrm{dd}, J=8.7,8.7 \mathrm{~Hz}), 2.73(1 \mathrm{H}$, ddd, $J=3.5,5.5,9.5 \mathrm{~Hz}), 2.84(1 \mathrm{H}, \mathrm{dd}, J=6.5,12.5 \mathrm{~Hz}), 2.92(1 \mathrm{H}, \mathrm{dd}, J=1.5,9.5 \mathrm{~Hz})$, $3.98(1 \mathrm{H}, \mathrm{dd}, J=9.0,11.0 \mathrm{~Hz}), 4.21(1 \mathrm{H}, \mathrm{dd}, J=5.0,11.0 \mathrm{~Hz}), 5.27(1 \mathrm{H}, \mathrm{ddd}, J=5.0,8.0$ $\mathrm{Hz}) \mathrm{ppm} .-{ }^{13} \mathrm{C}$ NMR $(126 \mathrm{MHz}): 16.5,21.06,36.1,36.3,36.4,39.5,40.2,55.4,66.5,122.6$, 138.3, 172.4, 213.7 ppm. - IR: $3419 \mathrm{~cm}^{-1}$, 2927, 2858, 1734, 1701, 1456, 1259, 1150, 1058, $837,707$. 


\section{(4aR,11aR,E)-7-Methyl-11-methylene-4,4a,5,6,9,10,11,11a-octahydrocyclonona[c]pyran- 3(1H)-one (13) $^{9}$}

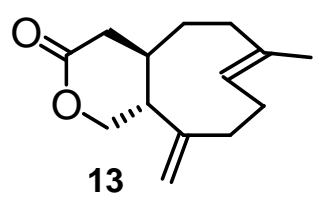

To a solution of methylenetriphenylphosphorane (58 $\mathrm{mg}, 0.22 \mathrm{mmol})$ in THF (3 mL) at $-10{ }^{\circ} \mathrm{C}$ was added a solution of $12(35 \mathrm{mg}, 0.158 \mathrm{mmol})$ in THF $(1.5 \mathrm{~mL})$ and the mixture was stirred at $-10{ }^{\circ} \mathrm{C}$ for $7 \mathrm{~h}$. Saturated aqueous solution of $\mathrm{NaHCO}_{3}(1 \mathrm{~mL})$, and dichloromethane $(10 \mathrm{~mL})$ were added at an interval of $5 \mathrm{~min}$. The mixture was dried over $\mathrm{Na}_{2} \mathrm{SO}_{4}$ and concentrated under reduced pressure. The residue was purified by column chromatography (hexane/ethyl acetate) to give lactone $13(21 \mathrm{mg}, 61 \%)$ as a colorless oil, solidifying at. $23{ }^{\circ} \mathrm{C}$ $[\alpha]_{\mathrm{D}}^{20}+149\left(\right.$ c 0.7 in $\left.\mathrm{CHCl}_{3}\right) ;$ lit: $^{9}[\alpha]_{\mathrm{D}}{ }^{27}+159\left(\right.$ c 0.7 in $\left.\mathrm{CHCl}_{3}\right) .-{ }^{1} \mathrm{H}$ NMR $(500 \mathrm{MHz}): 1.52-$ $1.59(\mathrm{~m}, 2 \mathrm{H}), 1.66(3 \mathrm{H}, \mathrm{d}, J=1.0 \mathrm{~Hz}), 1.92(1 \mathrm{H}, \mathrm{ddd}, J=2.0,6.0,10.0 \mathrm{~Hz}), 2.01-2.20(5 \mathrm{H}$, m), 2.32-2.35 (1 H, m), 2.35 (1 H, dd, $J=4.0,14.5 \mathrm{~Hz}), 2.41-2.49$ (1 H, m), 2.79 (1 H, dd, $J$ $=7.0,14.5 \mathrm{~Hz}), 3.87(1 \mathrm{H}, \mathrm{dd}, J=10.0,10.0 \mathrm{~Hz}), 4.12(1 \mathrm{H}, \mathrm{dd}, J=6.0,11.5 \mathrm{~Hz}), 4.89(1 \mathrm{H}$, s), 4.95 (1 H, s), 5.33 (br dd, $J=7.8,7.8 \mathrm{~Hz}$ ) ppm. $-{ }^{13} \mathrm{C}$ NMR (126 MHz): 16.3, 24.7, 35.2, 36.9, 37.2, 39.7, 40.7, 48.3, 70.7, 112.6, 124.3, 135.5, 152.7, 173.5 ppm. - IR: $2924 \mathrm{~cm}^{-1}$, $2855,1750,1329,1151,1076,895,732$.

\section{4-Methylpent-(2E)-en-1-ol ${ }^{10}$}

To a solution of 3-methylbut-1-yne (8.58 g, $0.126 \mathrm{~mol}, 12.6 \mathrm{~mL})$ in

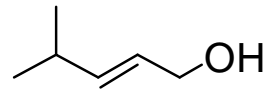

THF $(160 \mathrm{~mL})$ was added at $-78^{\circ} \mathrm{C}$ a $2.5 \mathrm{M}$ solution of $\mathrm{BuLi}$ in hexane $(0.126 \mathrm{~mol}, 50.6 \mathrm{~mL})$ dropwise over $10 \mathrm{~min}$. The reaction mixture was stirred at $-78{ }^{\circ} \mathrm{C}$ for $40 \mathrm{~min}$, then dry paraformaldehyde $(5.4 \mathrm{~g}, 0.18 \mathrm{~mol})$ was added, and the mixture was allowed to warm up to $23{ }^{\circ} \mathrm{C}$, and then stirred at $23{ }^{\circ} \mathrm{C}$ for $16 \mathrm{~h}$. Saturated aqueous $\mathrm{NH}_{4} \mathrm{Cl}$ solution (5 $\mathrm{mL}$ ) was added, followed by diethyl ether $(200 \mathrm{~mL})$. The mixture was dried over $\mathrm{Na}_{2} \mathrm{SO}_{4}$, filtered through a plug of Celite and concentrated under reduced pressue at $23{ }^{\circ} \mathrm{C}$ The residue was distilled in vacuum (80 mbar) to give 4-methylpent-2-yn-1-ol ${ }^{10}(11.5 \mathrm{~g}, 93 \%)$ as a colorless oil (b.p. $\left.105{ }^{\circ} \mathrm{C} / 80 \mathrm{mbar}\right)$. This was dissolved in THF $(40 \mathrm{~mL})$ and added dropwise within $10 \mathrm{~min}$ to a $2 \mathrm{M}$ solution of $\mathrm{LiAlH}_{4}$ in THF $(0.120 \mathrm{~mol}, 60 \mathrm{~mL})$. The mixture was then heated at reflux for $10 \mathrm{~h}$. Saturated aqueous $\mathrm{NaOH}(10 \mathrm{~mL})$ was added dropwise slowly at 0 ${ }^{\circ} \mathrm{C}$, followed by diethyl ether $(200 \mathrm{~mL})$. The resulting heterogeneous mixture was stirred at 23 
${ }^{\circ} \mathrm{C}$ for $2 \mathrm{~h}$, then dried over $\mathrm{Na}_{2} \mathrm{SO}_{4}$ and concentrated under reduced pressure at $23{ }^{\circ} \mathrm{C}$ The residue was distilled in vacuo (80 mbar) to give the product as a colorless oil $(10.1 \mathrm{~g}, 86 \%)$. B.p. $95{ }^{\circ} \mathrm{C} / 80$ mbar. ${ }^{1} \mathrm{H}$ NMR $(500 \mathrm{MHz}): 1.00(6 \mathrm{H}, \mathrm{d}, J=6.5 \mathrm{~Hz}), 1.27(1 \mathrm{H}, \mathrm{dd}, J=5.5,5.5$ Hz), $2.32(1 \mathrm{H}$, sextet, $J=6.5 \mathrm{~Hz}), 4.09(2 \mathrm{H}, \mathrm{dd}, J=5.9,5.9 \mathrm{~Hz}), 5.59(1 \mathrm{H}$, ddd, $J=5.9,5.9$, $15.5 \mathrm{~Hz}), 5.66(1 \mathrm{H}, \mathrm{dd}, J=5.9,15.5 \mathrm{~Hz}) \mathrm{ppm}$.

\section{1-Iodo-4-methylpent-(2E)-ene (14)}

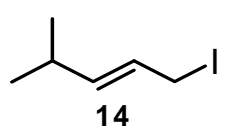

To a solution of triphenylphosphine $(0.66 \mathrm{~g}, 2.5 \mathrm{mmol})$ and imidazole $(215$ $\mathrm{mg}, 3.16 \mathrm{mmol})$ in dichloromethane $(6.5 \mathrm{~mL})$ was added at $0{ }^{\circ} \mathrm{C}$ iodine (690 mg, $2.7 \mathrm{mmol}$ ) in 3 portions, followed by a solution of 4-methylpent(2E)-en-1-ol (200 mg, $2.3 \mathrm{mmol})$ in dichloromethane $(5 \mathrm{~mL})$ within $5 \mathrm{~min}$. The reaction mixture was stirred at $0{ }^{\circ} \mathrm{C}$ for $1 \mathrm{~h}$. It was diluted with saturated aqueous solution of $\mathrm{Na}_{2} \mathrm{~S}_{2} \mathrm{O}_{3}$ $(30 \mathrm{~mL})$. The mixture was stirred for $20 \mathrm{~min}$ at $23{ }^{\circ} \mathrm{C}$, then extracted with dichloromethane (3 $\times 10 \mathrm{~mL}$ ). The combined organic extracts were dried over $\mathrm{Na}_{2} \mathrm{SO}_{4}$ and $4 \AA$ molecular sieves, and concentrated under reduced pressure at $23{ }^{\circ} \mathrm{C}$ The crude allyl iodide 14 isolated as a light yellow oil [ ${ }^{1} \mathrm{H}$ NMR (500 MHz): 0.98 (6 H, d, $\left.J=7.0 \mathrm{~Hz}\right), 2.25-2.34$ (1 H, m), 3.85-3.89 (2 $\mathrm{H}, \mathrm{m}), 5.65-5.69(2 \mathrm{H}, \mathrm{m}) \mathrm{ppm}$.] was immediately used in the next step.

\section{Coraxeniolide A (3)}

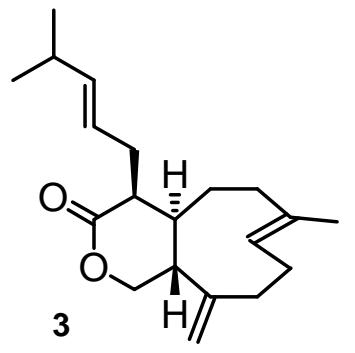

To a solution of lactone $13(48 \mathrm{mg}, 0.22 \mathrm{mmol})$ in THF $(0.5 \mathrm{~mL})$ and DMPU $(0.3 \mathrm{~mL})$ was added at $-78^{\circ} \mathrm{C}$ a $1 \mathrm{M}$ solution of LHMDS in THF $(0.25 \mathrm{~mol}, 0.25 \mathrm{~mL})$. The mixture was stirred at $-78{ }^{\circ} \mathrm{C}$ for 30 $\mathrm{min}$, then a solution of 1-iodo-4-methylpent-(2E)-ene $(0.44 \mathrm{mmol}, 87$ $\mu \mathrm{L})$ in THF $(0.2 \mathrm{~mL})$ was added, and the mixture was stirred at $-40{ }^{\circ} \mathrm{C}$ for $20 \mathrm{~h}$. The reaction was quenched by adding saturated aqueous $\mathrm{NaHCO}_{3}$ solution $(0.5 \mathrm{~mL})$. Dichloromethane $(5 \mathrm{~mL})$ and $\mathrm{Na}_{2} \mathrm{SO}_{4}$ were added, and the organic phase was concentrated under reduced pressure. The mixture of coraxeniolide A (3) and 4-epi-3 (47 mg, dr 1:6) was separated from unreacted starting material 13 (9 mg) by column chromatography (hexane/EtOAc $\left.+0.5 \% \mathrm{Et}_{3} \mathrm{~N}\right)$, dissolved in toluene $(1 \mathrm{~mL})$ and 
phosphazene base $\mathrm{P}_{2}$-Et (108 $\mathrm{mg}, 0.32 \mathrm{mmol}$ ) was added. The mixture was stirred at $23{ }^{\circ} \mathrm{C}$ for $22 \mathrm{~h}$, then separated by column chromatography (hexane/EtOAc $+0.5 \% \mathrm{Et}_{3} \mathrm{~N}$ ) to give coraxeniolide A (3) (33 mg) and 4-epi-3 (8 mg) as colorless oils.

3: $[\alpha]_{\mathrm{D}}{ }^{20}+71$ (c 0.40 in $\left.\mathrm{CHCl}_{3}\right)$; lit: ${ }^{9}[\alpha]_{\mathrm{D}}{ }^{22}+72\left(\right.$ c 0.47 in $\left.\mathrm{CHCl}_{3}\right) \cdot-{ }^{1} \mathrm{H} \mathrm{NMR}(500 \mathrm{MHz})$ : $0.97(6 \mathrm{H}, \mathrm{dd}, J=1.8,6.9 \mathrm{~Hz}), 1.03(1 \mathrm{H}, \mathrm{ddd}, J=3.0,3.5,13.5 \mathrm{~Hz}), 1.67(3 \mathrm{H}, \mathrm{d}, J=1.0$ $\mathrm{Hz}), 1.71(1 \mathrm{H}$, ddd, $J=3.5,3.5,14.0 \mathrm{~Hz}), 1.92(1 \mathrm{H}, \mathrm{td}, J=4.5,12.5 \mathrm{~Hz}), 1.96-2.02(1 \mathrm{H}$, m), 2.03-2.05 (1 H, m), 2.05-2.07 (1 H, m), 2.07-2.11 (1 H, m), 2.14-2.16 (1 H, m), 2.18$2.22(1 \mathrm{H}, \mathrm{m}), 2.24(1 \mathrm{H}, \mathrm{dd}, J=7.0,13.5 \mathrm{~Hz}), 2.30-2.35(1 \mathrm{H}, \mathrm{m}), 2.43-2.48(1 \mathrm{H}, \mathrm{m}), 2.52-$ $2.60(1 \mathrm{H}, \mathrm{m}), 2.77-2.84(1 \mathrm{H}, \mathrm{m}, J=6.0,14.0 \mathrm{~Hz}), 3.90(1 \mathrm{H}, \mathrm{t}, J=12.0 \mathrm{~Hz}), 4.16(1 \mathrm{H}, \mathrm{dd}$, $J=7.0,12.0 \mathrm{~Hz}), 4.95(1 \mathrm{H}, \mathrm{s}), 4.96(1 \mathrm{H}, \mathrm{s}), 5.28-5.35(2 \mathrm{H}, \mathrm{m}), 5.48(1 \mathrm{H}, \mathrm{dd}, J=6.5,15.5$ Hz) ppm. $-{ }^{13} \mathrm{C}$ NMR (126 MHz): 16.4, 22.6, 25.0, 29.7, 29.9, 31.1, 35.6, 39.6, 42.7, 44.1, 49.6, 70.6, 112.1, 123.3, 123.8, 136.0, 140.6, 153.0, 175.2 ppm. - IR: $3067 \mathrm{~cm}^{-1}, 2928,1749$, 1437, $1100,971$.

4-epi-3: $[\alpha]_{\mathrm{D}}{ }^{20}-27.5$ (c 0.2 in $\mathrm{CHCl}_{3}$ ); lit: ${ }^{9}[\alpha]_{\mathrm{D}}{ }^{22}-25.6$ (c 0.22 in $\mathrm{CHCl}_{3}$ ). $-{ }^{1} \mathrm{H} \mathrm{NMR}(500$ MHz): $0.96(6 \mathrm{H}, \mathrm{dd}, J=1.5,7.0 \mathrm{~Hz}), 1.40-1.51(1 \mathrm{H}, \mathrm{m}), 1.66(3 \mathrm{H}, \mathrm{br} \mathrm{s}), 1.71-1.73(1 \mathrm{H}$, m), 1.75-1.78 (1 H, m), 1.83 (1 H, ddd, $J=7.5,10.0,10.0 \mathrm{~Hz}), 2.00-2.06$ (3 H, m), 2.18 (1 $\mathrm{H}$, ddd, $J=3.5,3.5,12.5 \mathrm{~Hz}), 2.21-2.24(1 \mathrm{H}, \mathrm{m}), 2.25-2.30(2 \mathrm{H}, \mathrm{m}), 2.33(1 \mathrm{H}, \mathrm{dd}, J=7.0$, $13.5 \mathrm{~Hz}), 2.40-2.48(1 \mathrm{H}, \mathrm{m}), 2.50-2.55(1 \mathrm{H}, \mathrm{m}), 3.99(1 \mathrm{H}, \mathrm{dd}, J=2.5,11.0 \mathrm{~Hz}), 4.14(1 \mathrm{H}$, $\mathrm{dd}, J=3.0,11.0 \mathrm{~Hz}), 4.69(1 \mathrm{H}, \mathrm{s}), 4.87(1 \mathrm{H}, \mathrm{s}), 5.31(1 \mathrm{H}, \mathrm{br} \mathrm{t}, J=7.5 \mathrm{~Hz}), 5.44(1 \mathrm{H}, \mathrm{dd}, J$ $=5.5,15.5 \mathrm{~Hz}), 5.46-5.52(1 \mathrm{H}, \mathrm{m}) \mathrm{ppm} .-{ }^{13} \mathrm{C} \mathrm{NMR}(126 \mathrm{MHz}): 16.4,22.6,22.7,25.0$, $31.1,31.5,34.7,35.2,39.6,43.8,44.9,48.8,67.0,113.4,123.7,124.5,135.4,141 ., 152.7$, 175.0 ppm. - IR $3070 \mathrm{~cm}^{-1}, 2928,1745,1435,1103,971$.

\section{References}

1) Hajos, Z. G.; Parrish, D. R. Org. Synth., Coll. Vol. 7, p.363 (1990); Vol. 63, p.26 (1985).

2) Heathcock, C. H.; Davidsen, S. K.; Hug, K. T.; Flippin, L. A. J. Org. Chem. 1986, 51, 3027-3037.

3) Schlosser, M.; Jenny, T.; Guggisberg, Y. Synlett 1990, 11, 704.

4) Schmidbaur, H.; Stühler, H.; Vornberger, W. Chem. Ber. 1972, 105, 1084-1086. This original procedure was slightly modified by conducting the reaction at reflux and not room temperature.

5) Sevillano, L. G.; Caballero, E.; Tome, F.; Medarde, M.; San Feliciano, A. Tetrahedron 2002, 58, 10103-10112. 
6) Shankar, S.; Coates, R. M. J. Org. Chem. 1998, 63, 9177-9182.

7) Kaiser, R.; Lamparsky, D. Helv. Chim. Acta 1976, 59, 1803.

8) Barrero, A. F.; Molina, J.; Oltra, J. E.; Altarejos, J.; Barragan, A.; Lara, A.; Mergot, S. Tetrahedron 1985, 51, 3813-3822.

9) Renneberg, D.; Pfander, H.; Leumann, C. J. J. Org. Chem. 2000, 65, 9069-9079.

10) Hatch, L. F.; Li, T. P. J. Org. Chem. 1963, 28, 2400-2403. 


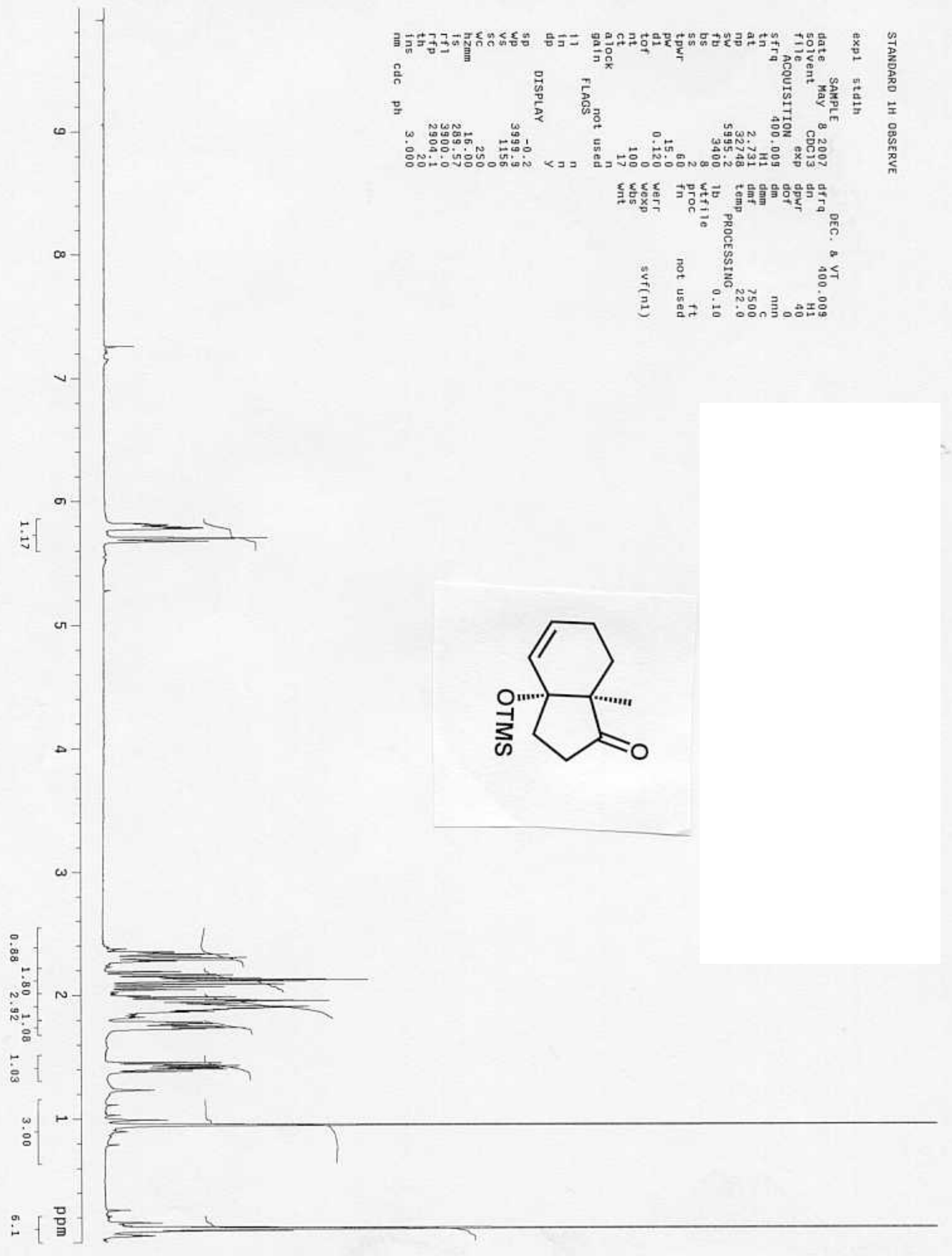




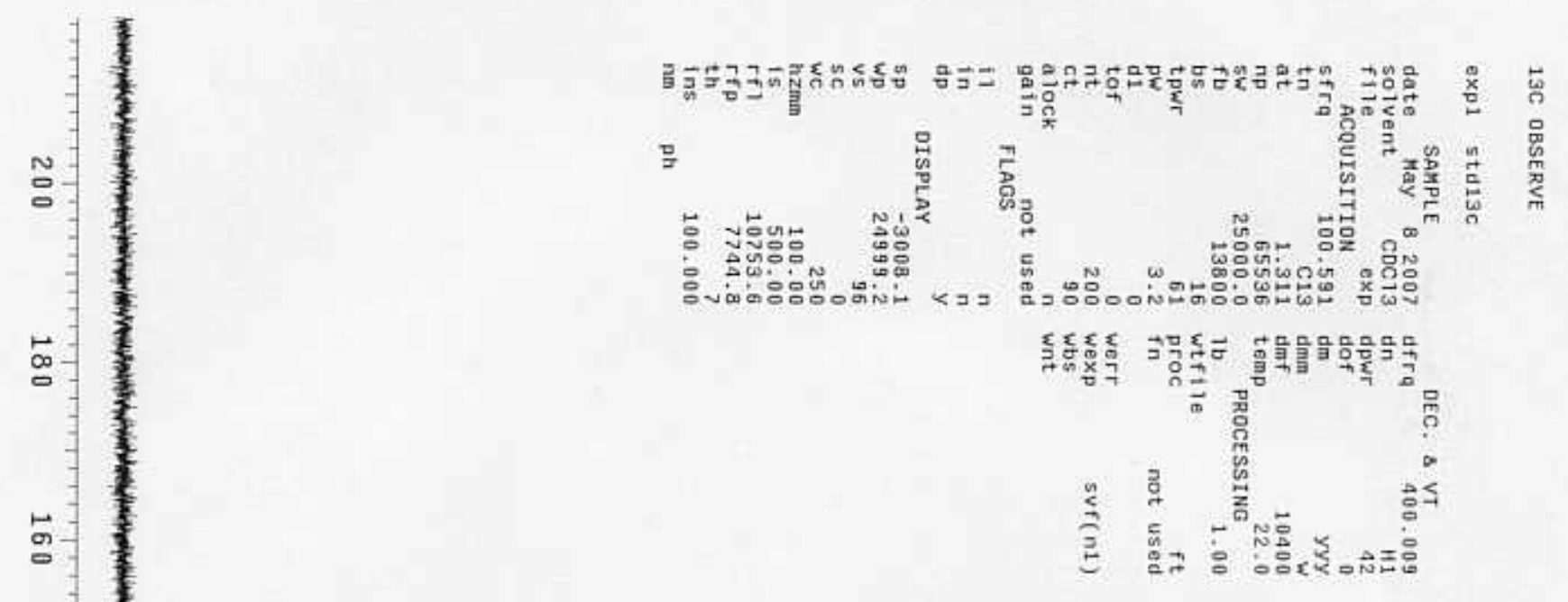

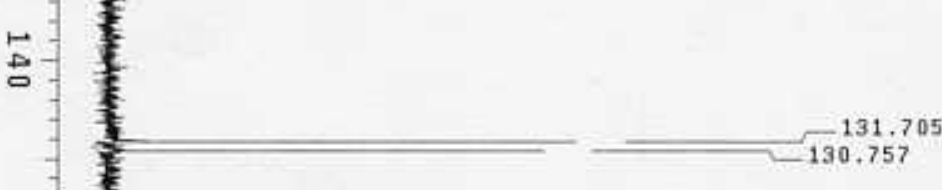

$\stackrel{5}{N}$

둥

-

-

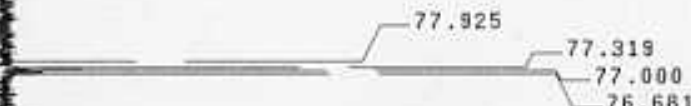

용

.52 .424

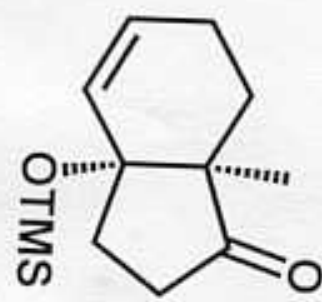

吕
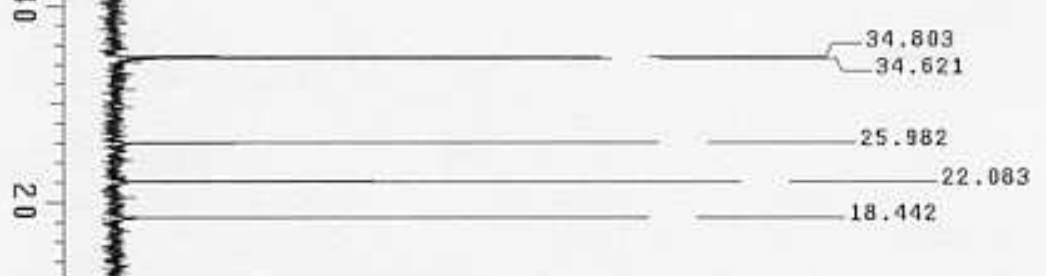

밈 


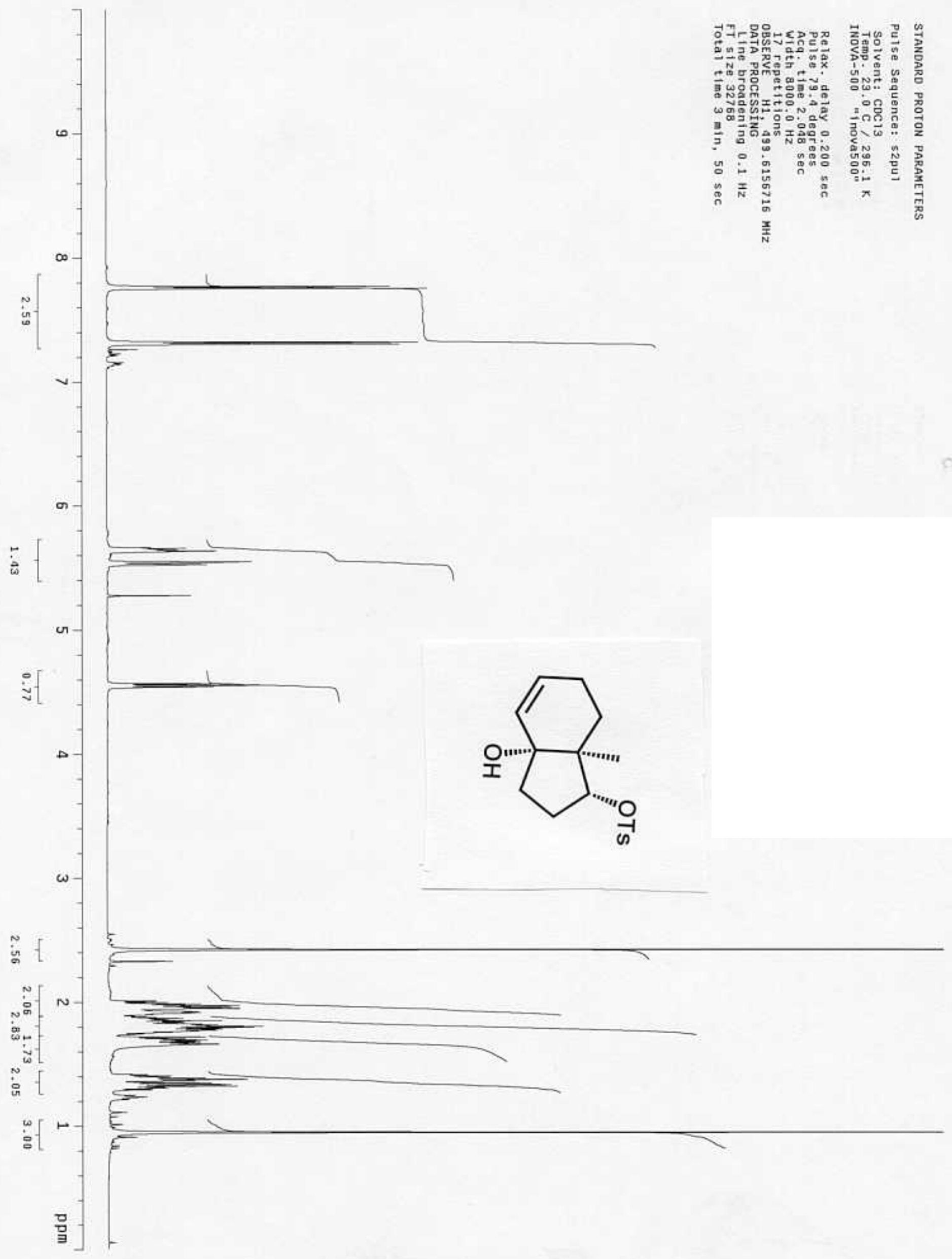




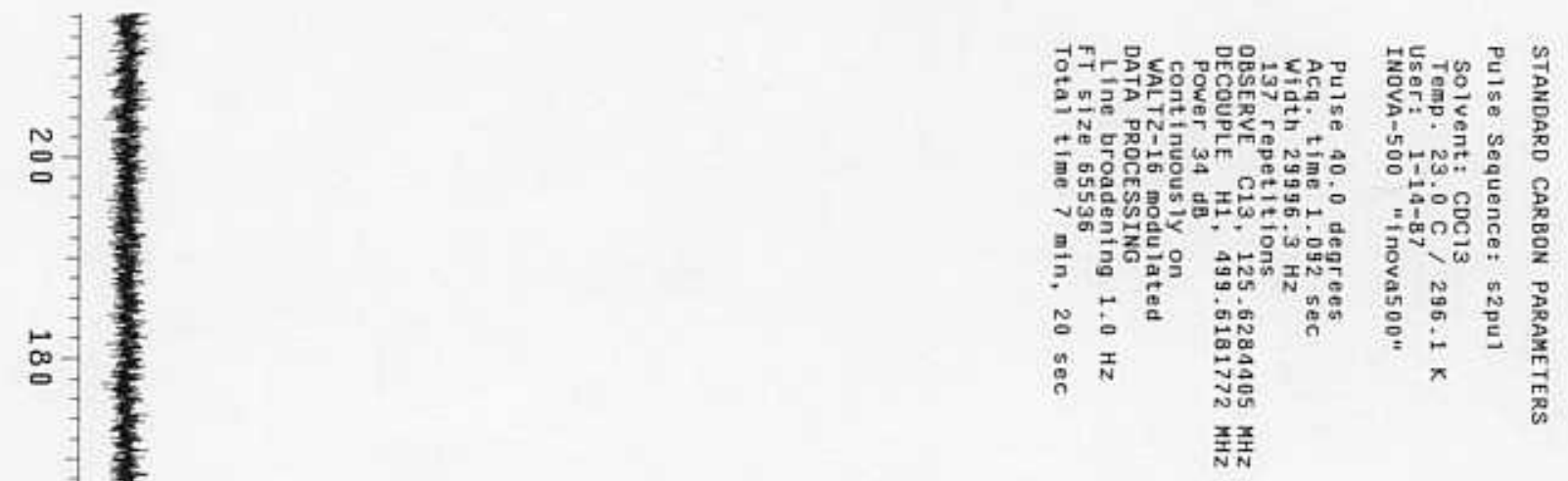

$-144.810$

点

$-134.295$

$-132.371$

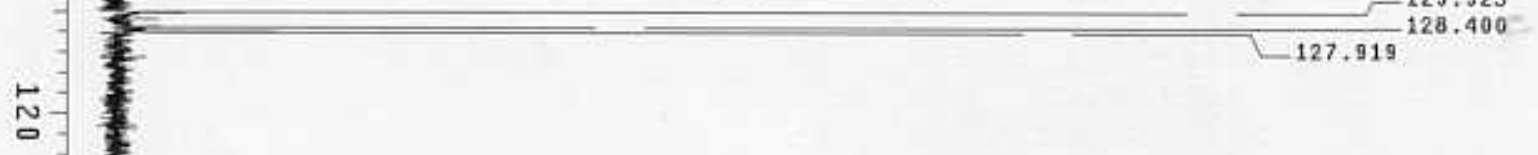

点
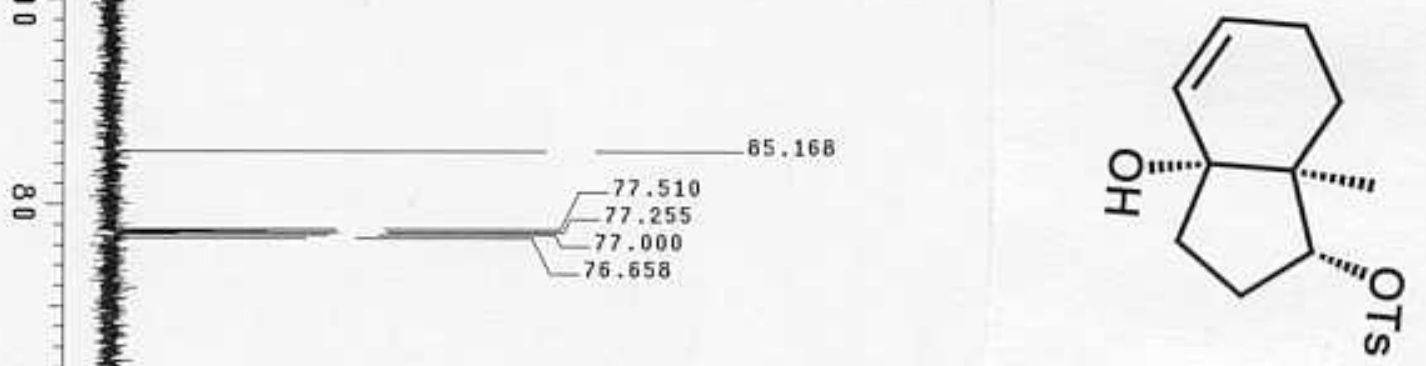

$-46.258$

b-

-

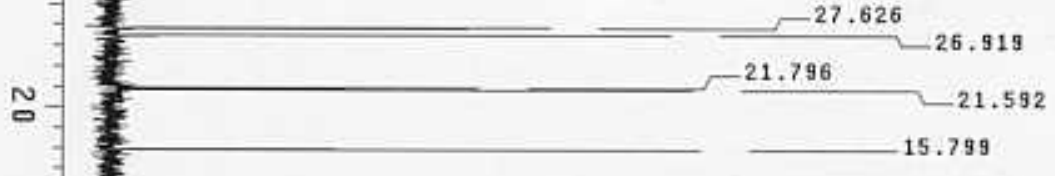

흡 


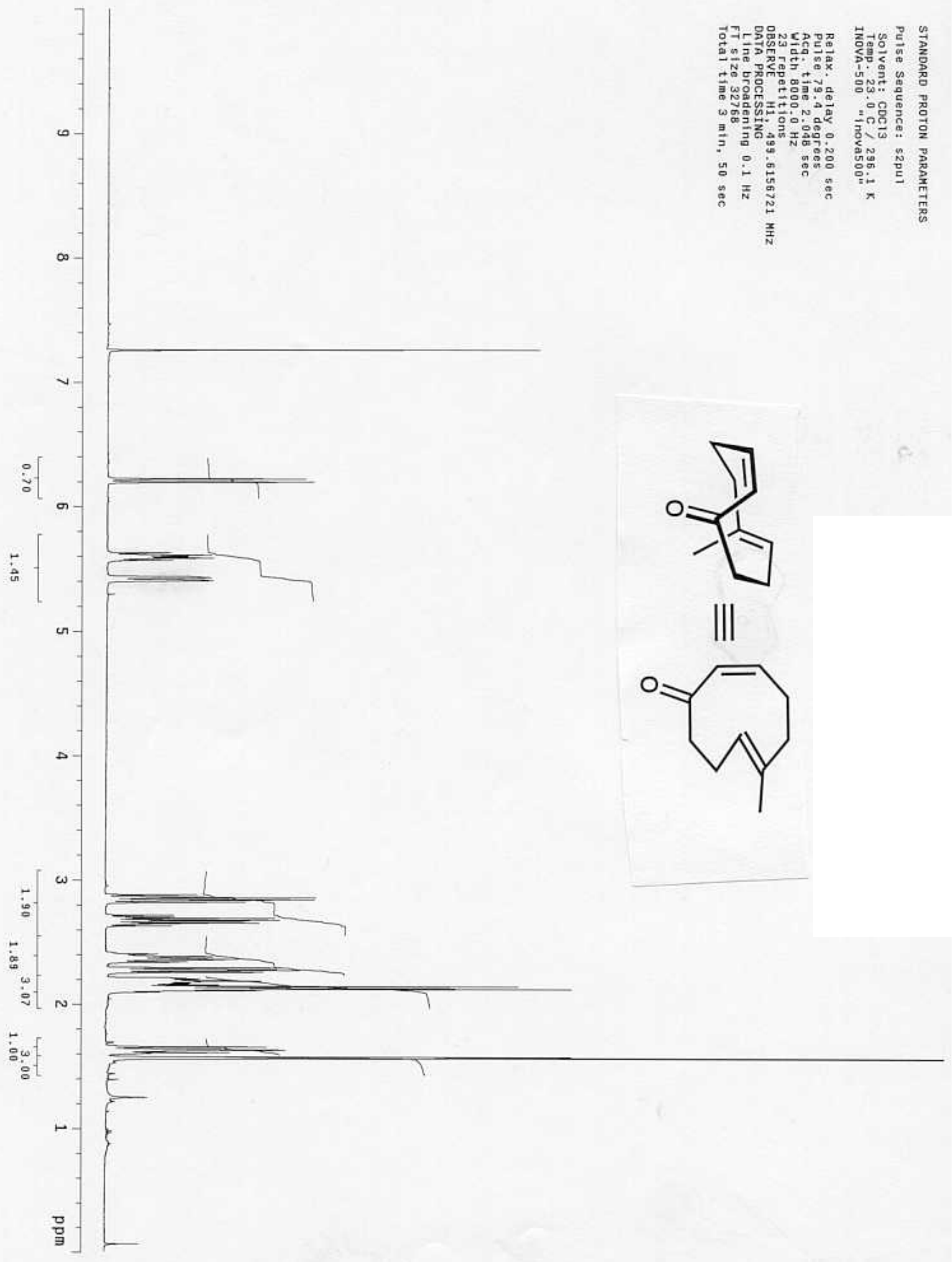



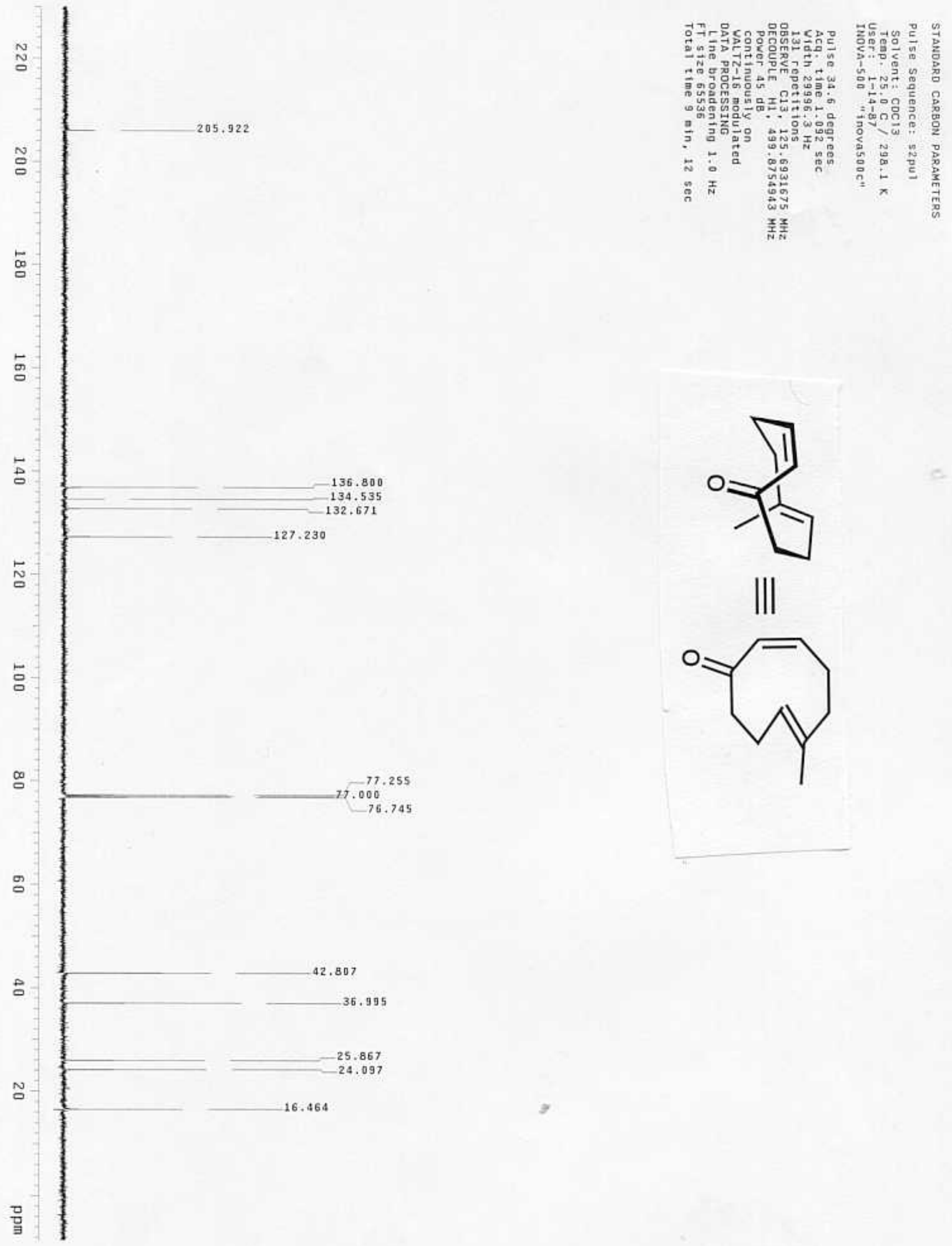


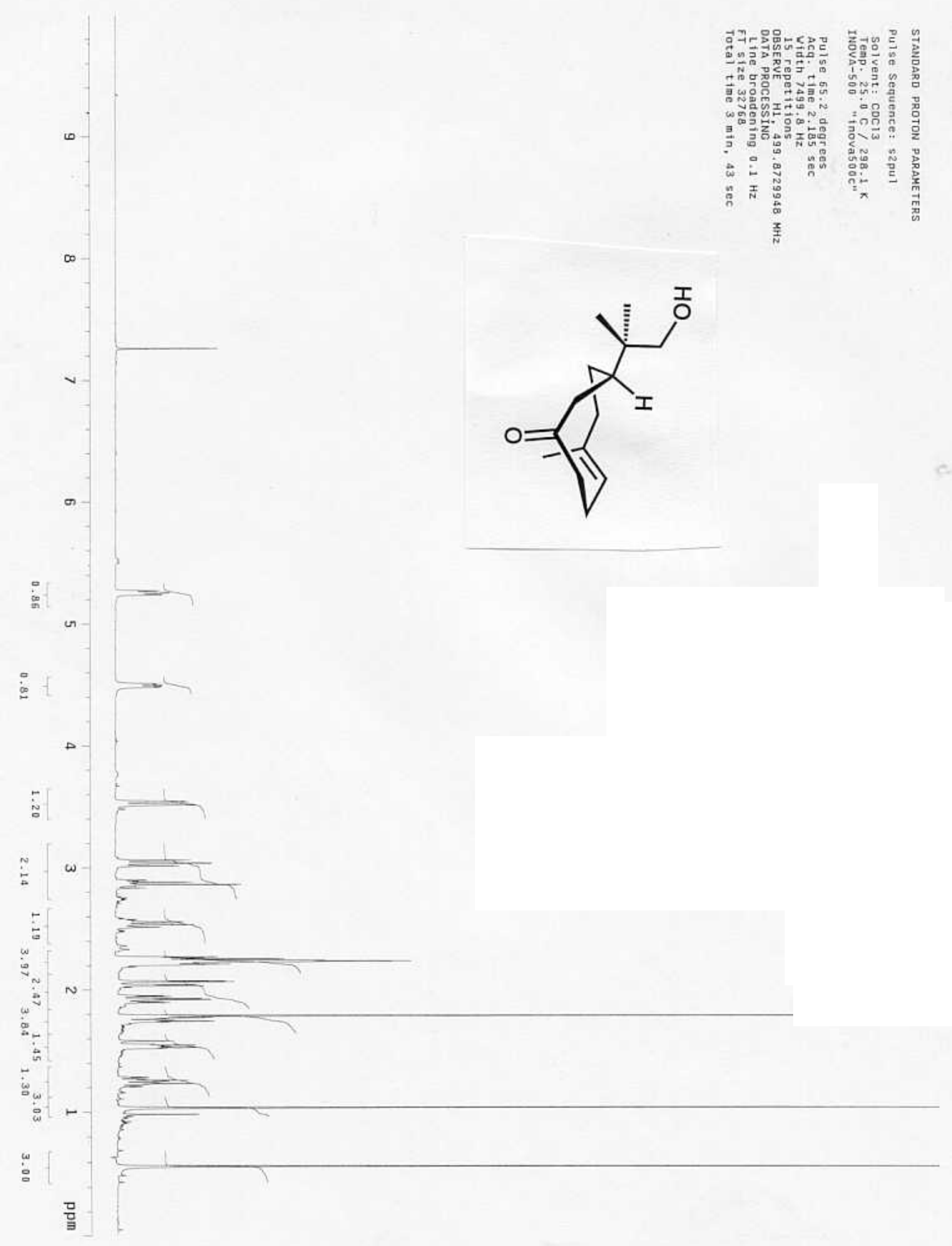



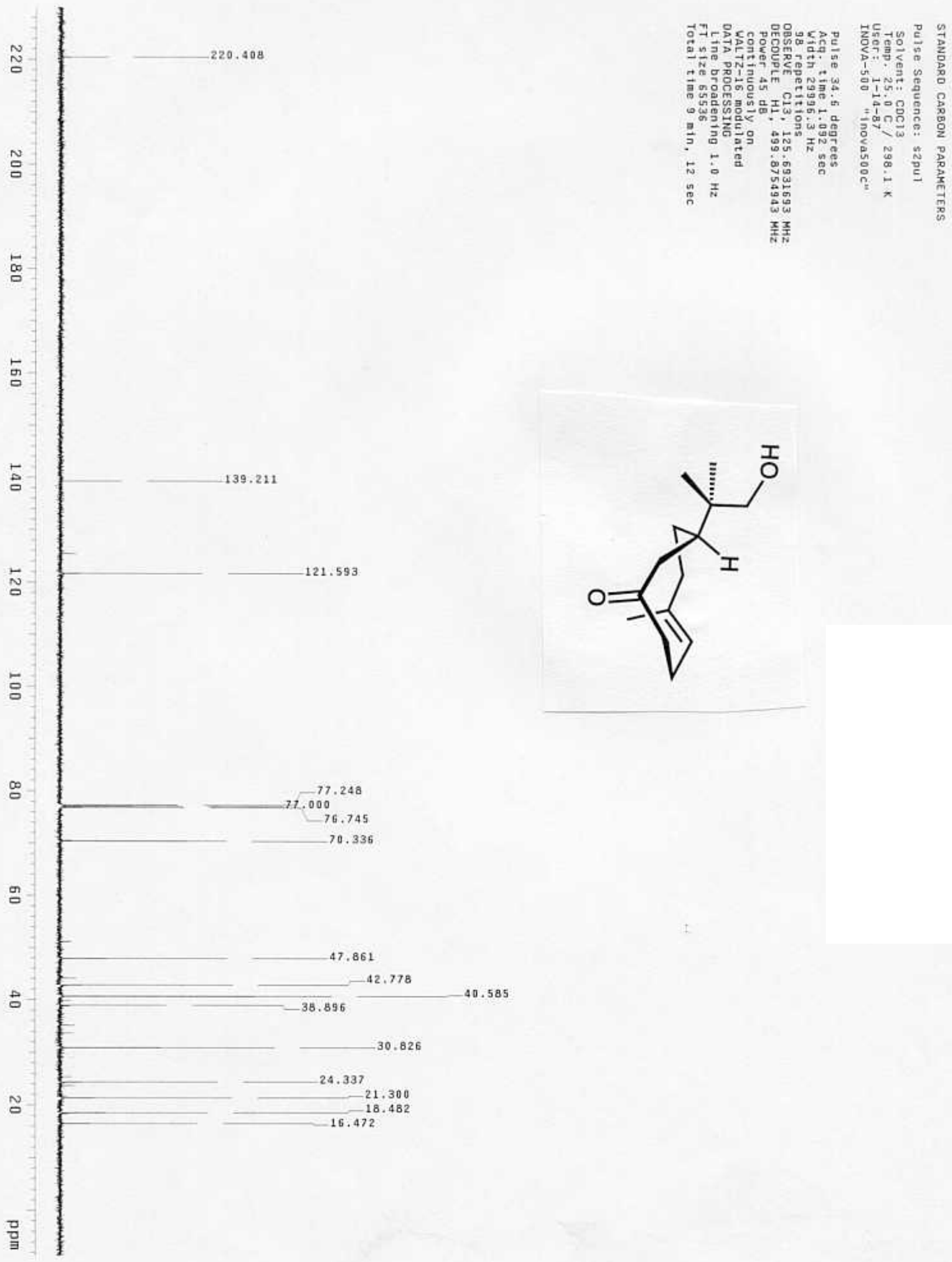


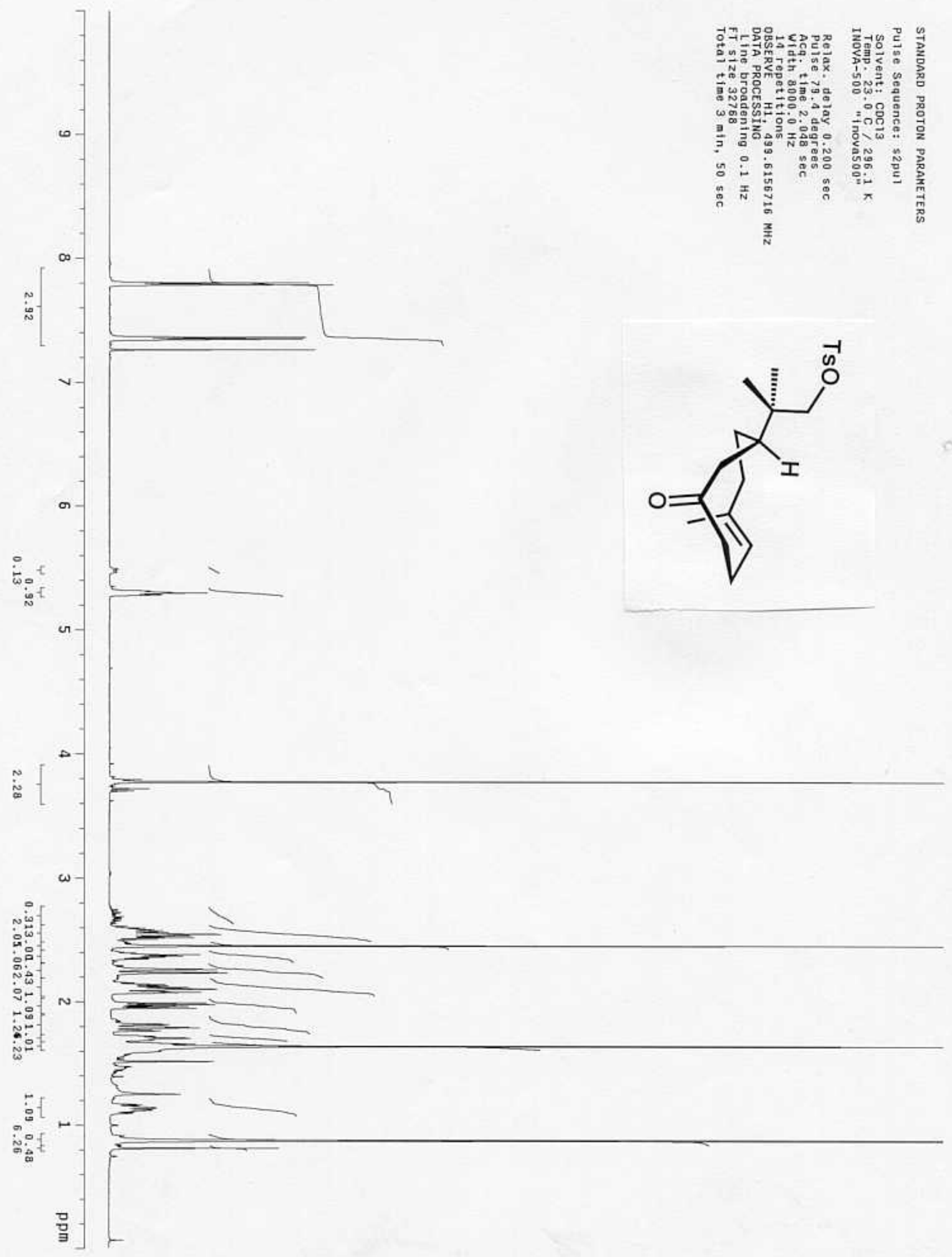




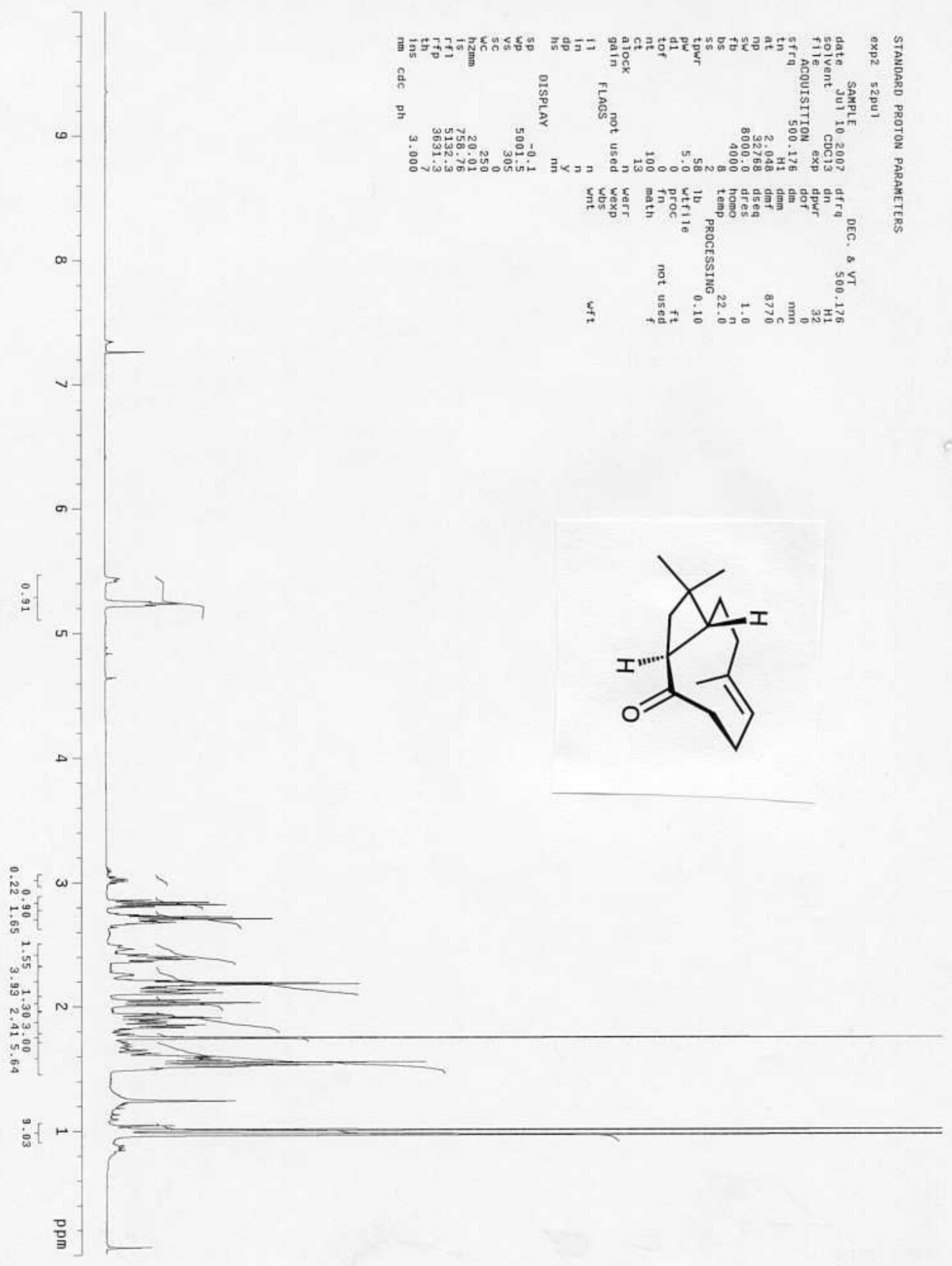



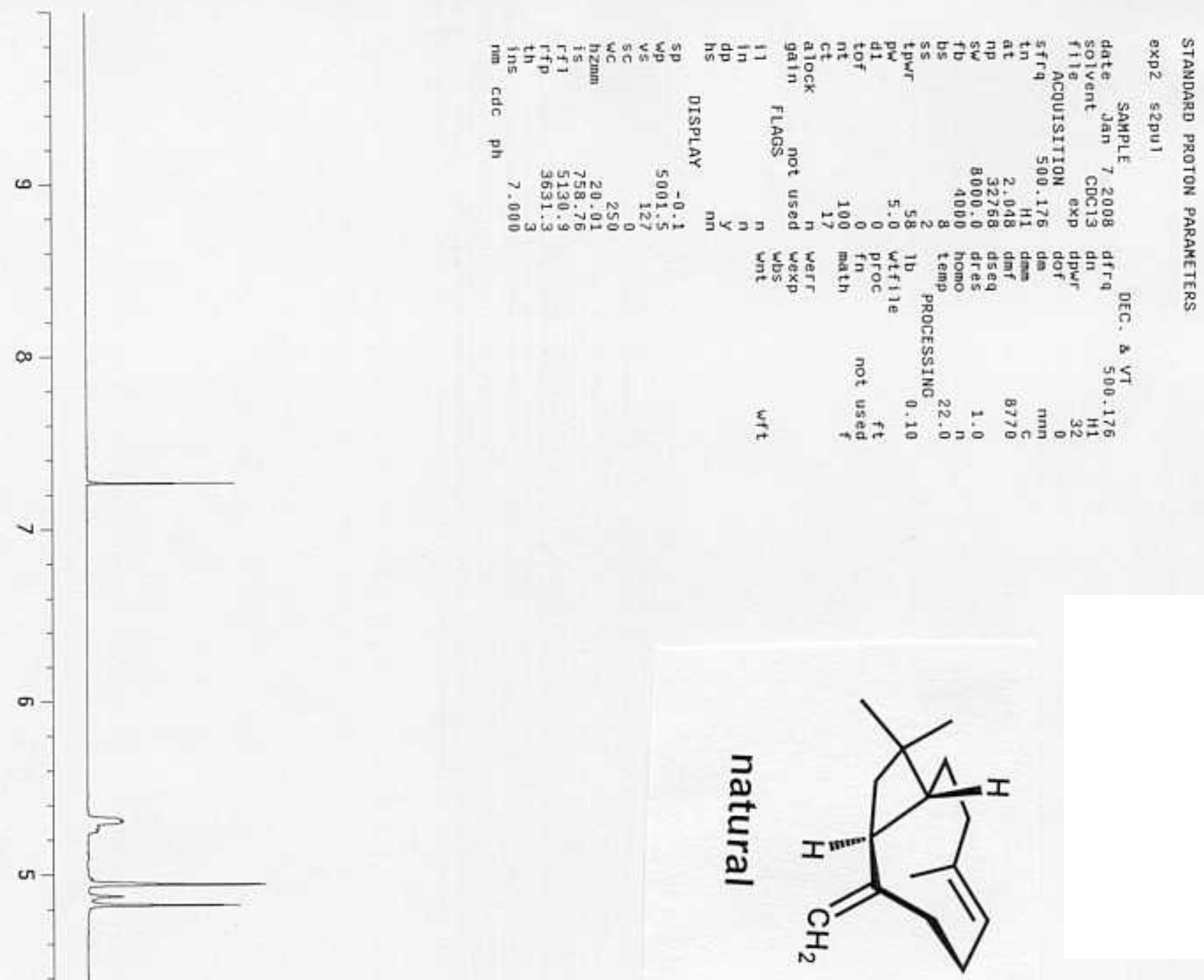


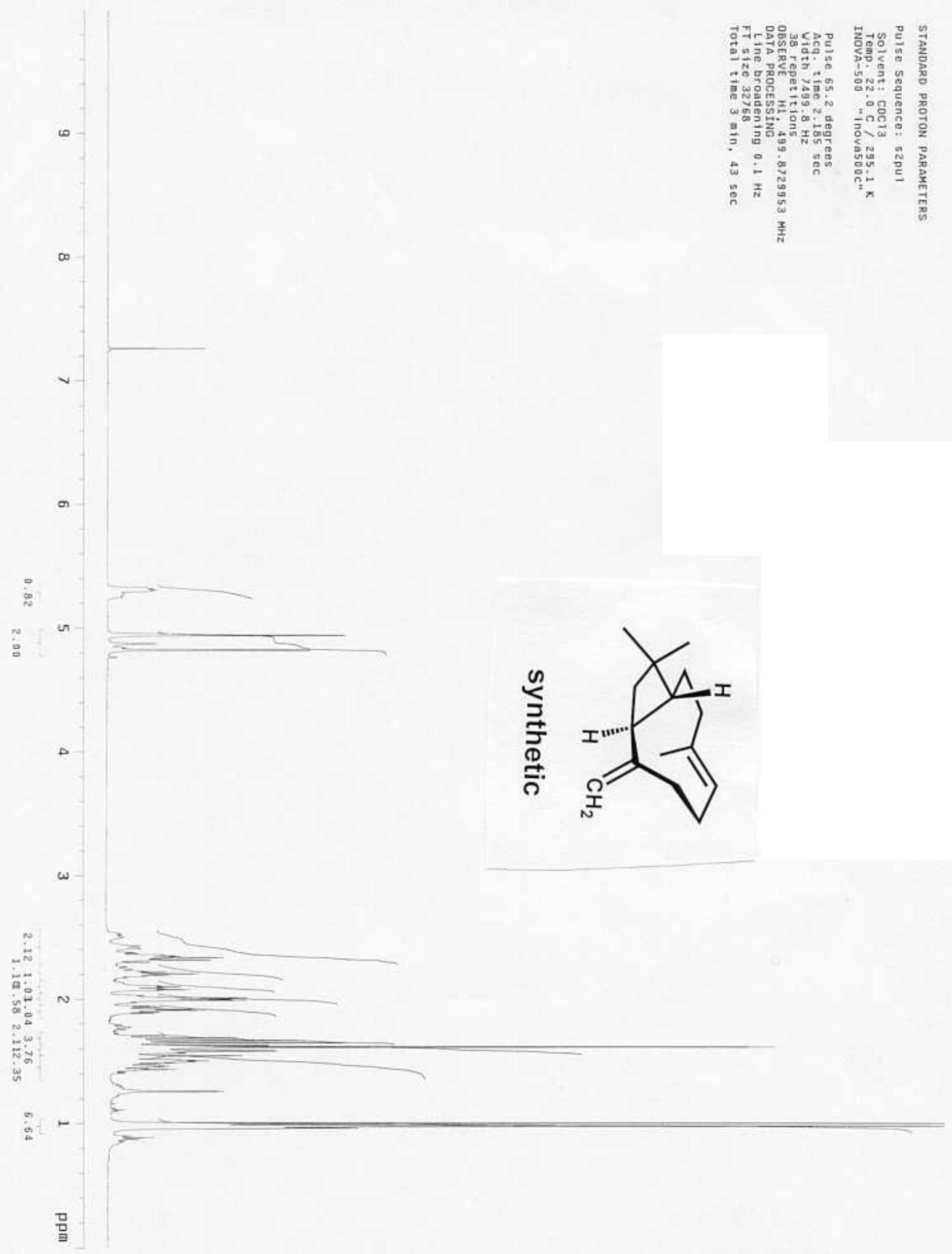




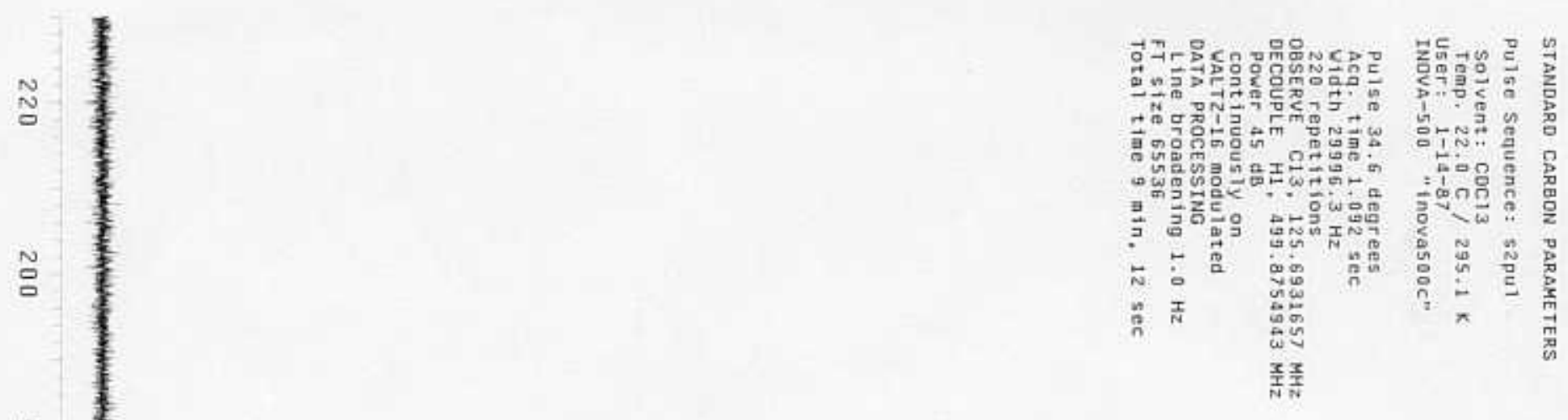

$-154.701$

$-124.288$

$-111.538$

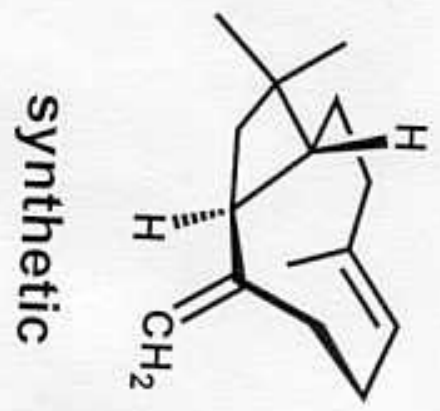

$\stackrel{\infty}{\circ}$

$-135.533$

N

요

$\infty$
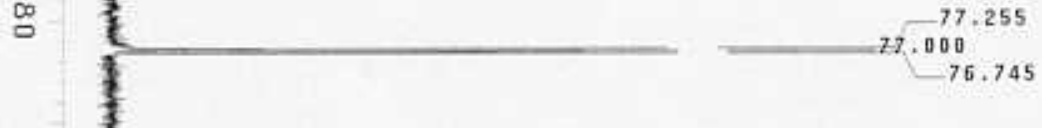

욤

웅

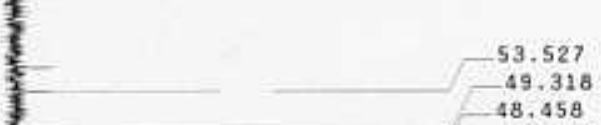

40.316

39,937
34,75

$-32.98$

$-29.821$

$-29.333$

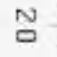

$-22.626$ 


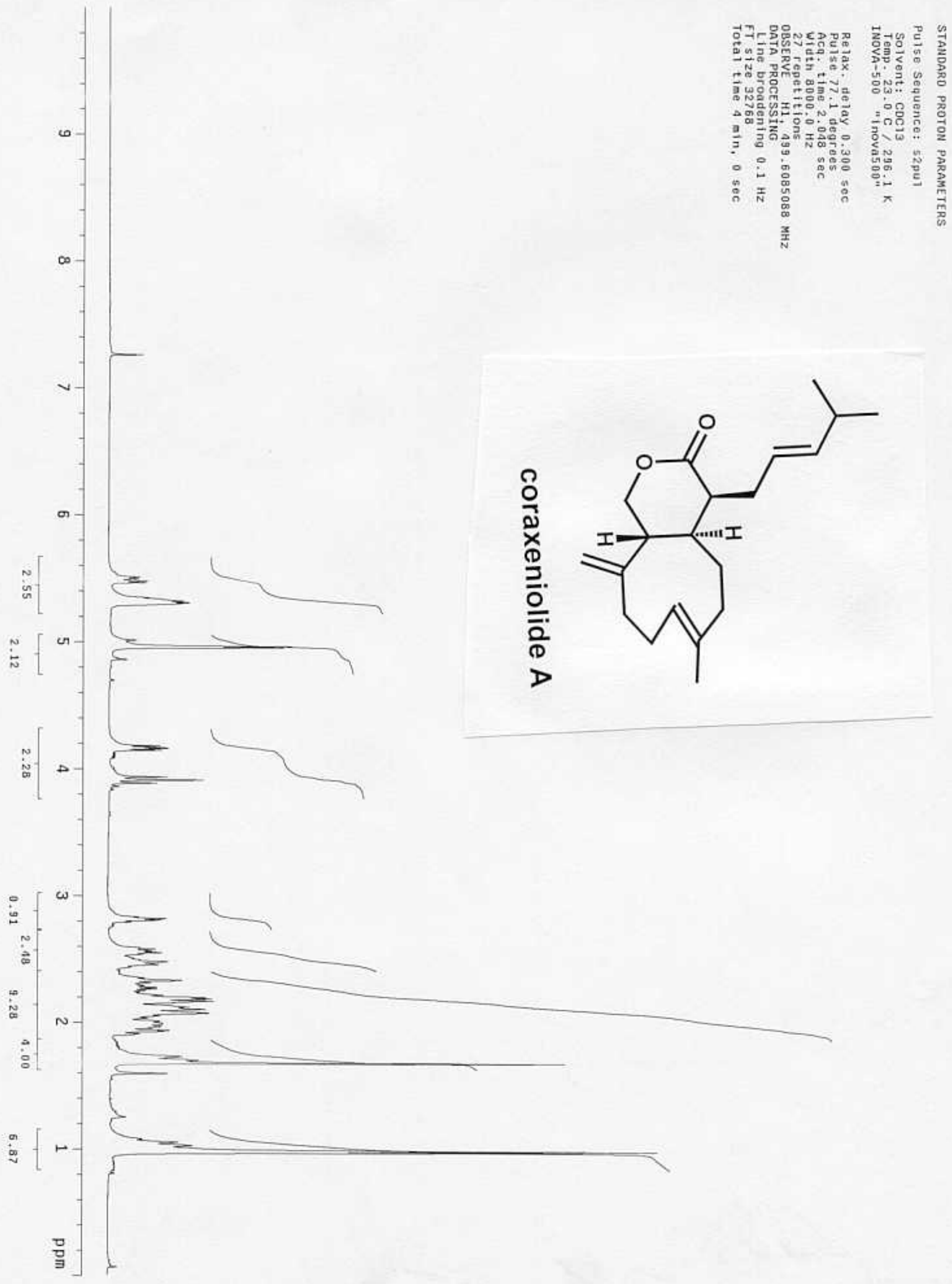




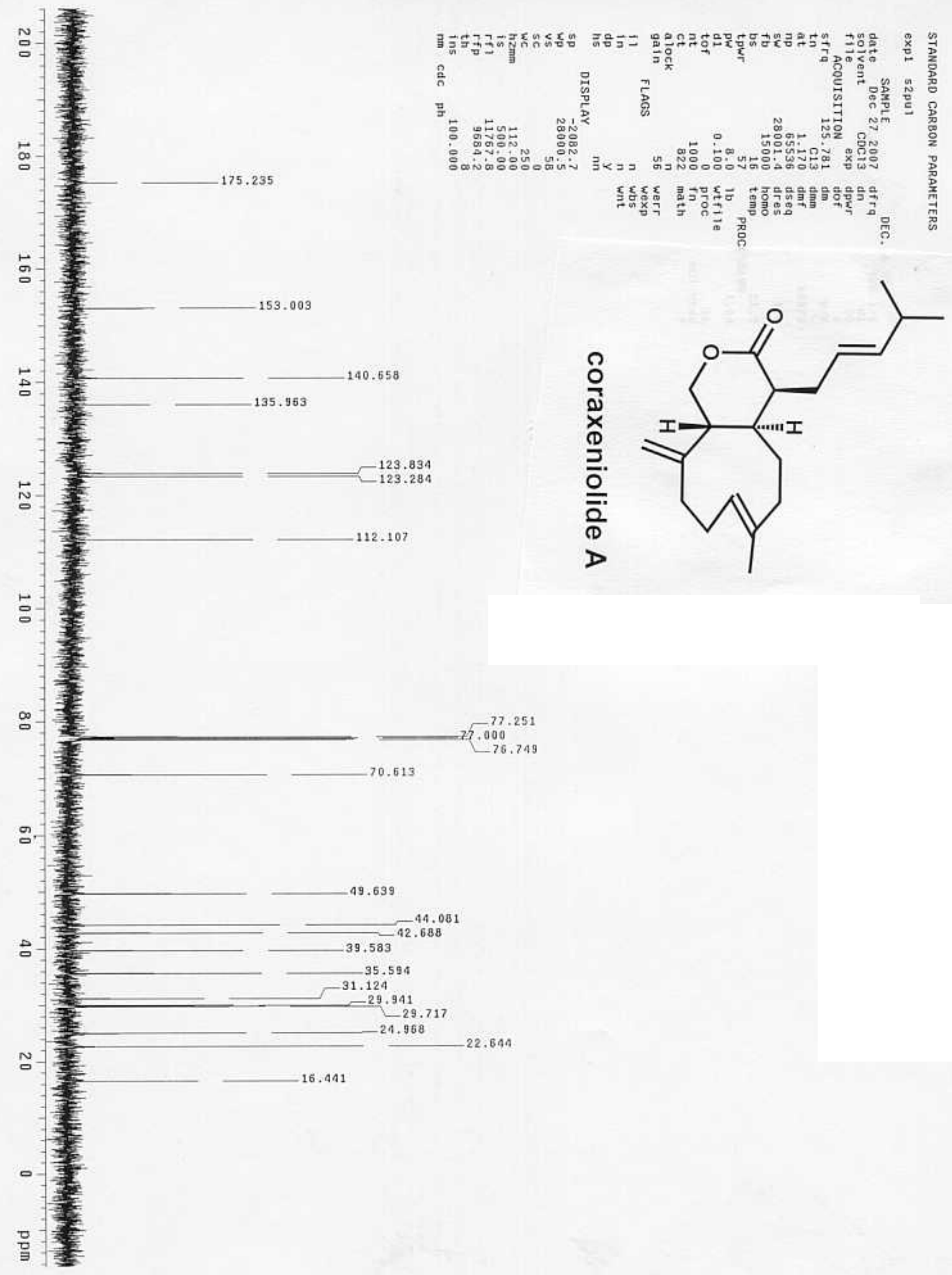




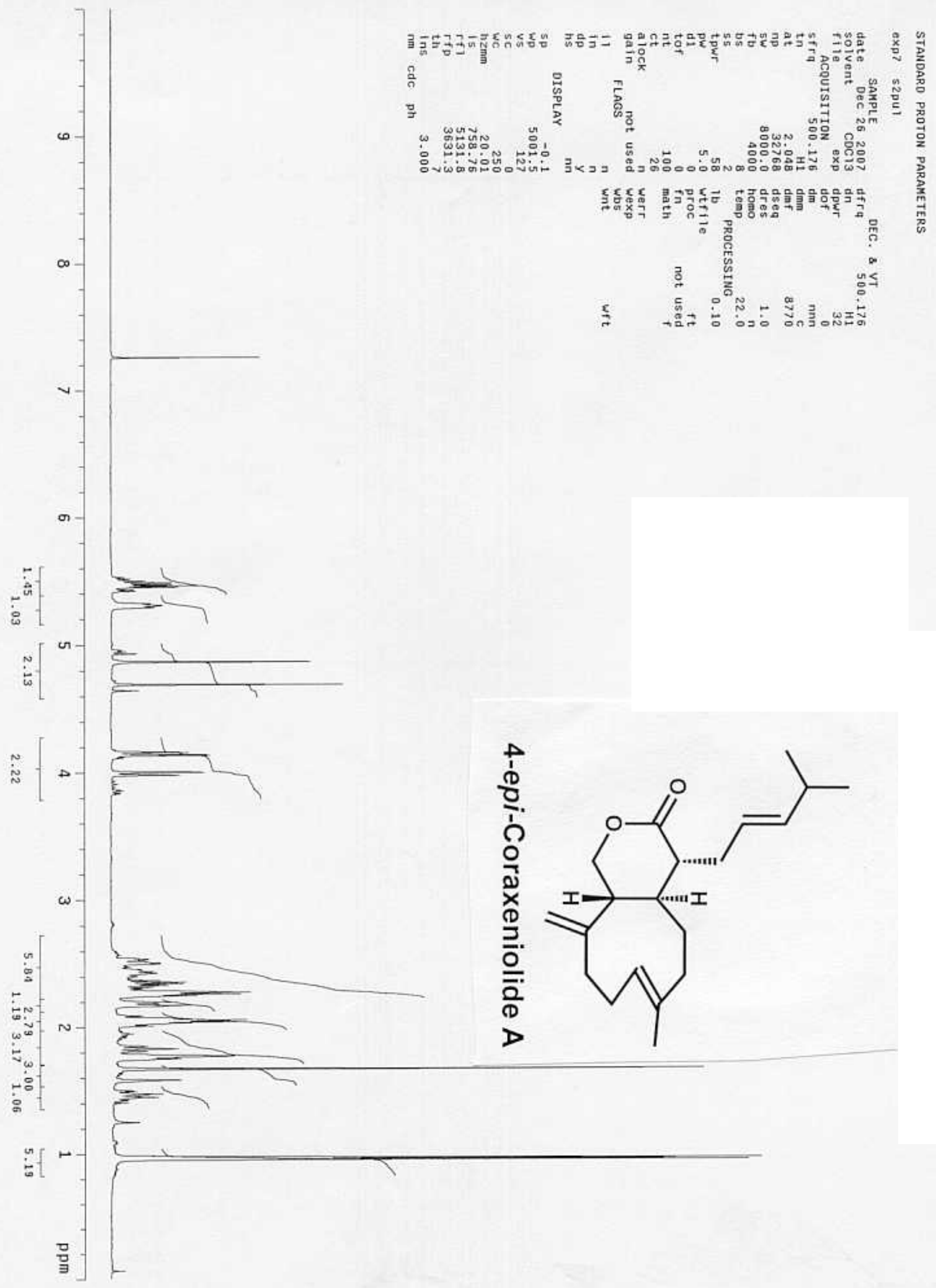




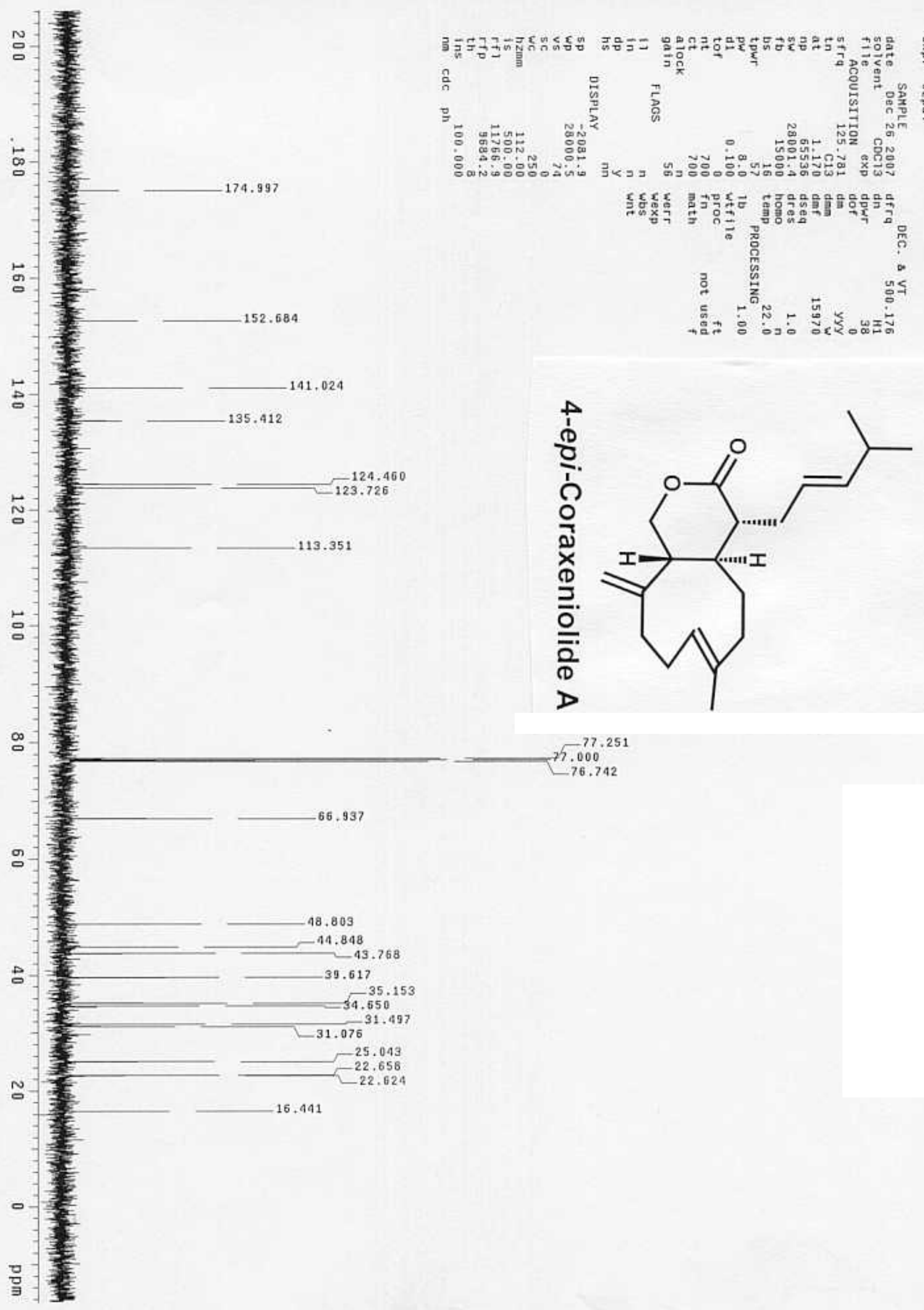

